\title{
Interpreting Deep Ensemble Learning through Radiologist Annotations for COVID-19 Detection in Chest Radiographs
}

\author{
Sivaramakrishnan Rajaraman ${ }^{1 *}$, Sudhir Sornapudi ${ }^{2}$, Philip O Alderson ${ }^{3}$, Les R Folio ${ }^{4}$, \\ Sameer K Antani ${ }^{1}$ \\ 1 Lister Hill National Center for Biomedical Communications, National Library of \\ Medicine, Maryland, Bethesda, United States of America \\ 2 Department of Electrical and Computer Engineering, Missouri University of Science \\ and Technology, Missouri, Rolla, United States of America \\ 3 School of Medicine, Saint Louis University, Missouri, St. Louis, United States of \\ America \\ 4 Radiological and Imaging Sciences, Clinical Center, National Institutes of Health, \\ Maryland, Bethesda \\ * email:sivaramakrishnan.rajaraman@nih.gov
}

\begin{abstract}
Data-driven deep learning (DL) methods using convolutional neural networks (CNNs) demonstrate promising performance in natural image computer vision tasks. However, using these models in medical computer vision tasks suffers from several limitations, viz., (i) adapting to visual characteristics that are unlike natural images; (ii) modeling random noise during training due to stochastic optimization and backpropagation-based learning strategy; (iii) challenges in explaining DL black-box behavior to support clinical decision-making; and (iv) inter-reader variability in the ground truth (GT) annotations affecting learning and evaluation. This study proposes a systematic approach to address these limitations for COVID-19 detection using chest X-rays (CXRs). Specifically, our contribution benefits from (i) pretraining specific to CXRs in transferring and fine-tuning the learned knowledge toward improving COVID-19 detection performance; (ii) using ensembles of the fine-tuned models to further improve performance compared to individual constituent models; (iii) performing statistical analyses at various learning stages to validate our claims; (iv) interpreting learned individual and ensemble model behavior through class-selective relevance mapping (CRM)-based region of interest (ROI) localization; (v) analyzing inter-reader variability and ensemble localization performance using Simultaneous Truth and Performance Level Estimation (STAPLE) methods. We observe that: (i) ensemble approaches improved classification and localization performance; and, (ii) inter-reader variability and performance level assessment helped guide algorithm design and parameter optimization. To the best of our knowledge, this is the first study to construct ensembles, perform ensemble-based disease ROI localization, and analyze inter-reader variability and algorithm performance for COVID-19 detection in CXRs.
\end{abstract}

\section{Introduction}

Coronavirus disease 2019 (COVID-19) is caused by the new Severe Acute Respiratory Syndrome Coronavirus 2 (SARS-CoV-2) that originated in Wuhan, China. The World Health Organization (WHO) declared this disease spread as an ongoing pandemic 1 . As of July 6, 2020, the pandemic has resulted in over 11 million cases, and more than 
530,000 deaths worldwide and continues to grow unabated. The disease commonly infects the lungs and results in pneumonia-like symptoms [2]. Reverse

transcription-polymerase chain reaction (RT-PCR) analysis is the gold standard to confirm infections. However, these tests are reported to exhibit varying sensitivity and are not widely available 2 . Radiological imaging using chest X-rays (CXRs) and computed tomography $(\mathrm{CT})$ scans, though not currently recommended in the United States, are commonly used radiological diagnostic support aids to manage COVID-19 disease progression [2]. While CT scans are more sensitive in detecting pulmonary disease manifestations than CXRs, their use is limited due to issues such as cross-contamination, non-portability, repeated sanitation requirements for CT examination rooms, and equipment, and exposing patients under investigation (PUI), hospital staff and technical personnel to the infection. Following the American College of Radiology (ACR) recommendations [3, CXRs are considered a viable alternative to CT scans in addressing some of these limitations. However, the pandemic nature of the disease has compounded the existing shortage of expert radiologists, particularly in third-world countries [4. Under these circumstances, artificial intelligence (AI) driven computer-aided diagnostic (CADx) tools have been considered as potentially viable alternatives for facilitating swift patient referrals or aiding appropriate medical care [5]. Several studies using data-driven deep learning (DL) algorithms with convolutional neural network (CNN) models in various strategies have been published for detecting, localizing, or measuring progression of COVID-19 using CXRs and CTs 4] 6. 7]. While there are scores of medical imaging CADx solutions that use DL approaches for disease detection including COVID-19, there are significant limitations in existing approaches related to data set type, size, scope, model architecture, and evaluation. We address these shortcomings and propose novel analyses to meet the urgent demand for COVID-19 detection using CXRs.

\section{Image modality-specific transfer learning}

Existing solutions tend to be disease-specific and require retraining on a large-collection of expert-annotated data to ensure use in real-world applications. Generalization of these approaches is challenged by available expert-annotations, their strength (i.e. weak image-level labels versus strong region of interest (ROI) localizing the pathology), and necessary computation resources. Under these circumstances, transfer learning strategies are commonly adopted 8 where the models are trained on a large-scale selection of stock photographic images like ImageNet 9 and then fine-tuned for the specific task. A problem with this approach is that the architecture and hyperparameters of these pre-trained models are optimized for natural image computer vision applications. In contrast, medical image collections bearing the desired pathology are significantly smaller in number. Therefore, using these models for medical visual analyses often results in a covariate shift and generalization issues due to the difference in source and target image modalities. Medical images are distinct in their characteristics such as highly localized disease ROIs, and varying appearances for the same disease label and severity [10]. Under these circumstances, the transferred knowledge from the natural image processing domain, while seemingly successful in disease classification, may not be optimal for disease localization. Medical images exhibit different visual characteristics than natural images through high intra-class variability and inter-class similarity, particularly for early-stage disease. To this end, we propose training DL models with suitable depth on a large-scale selection of medical images of the same modality to learn relevant feature representations that can be transferred and fine-tuned for related medical visual recognition tasks. Such medical modality-specific transfer learning could improve DL performance and generalization by learning the common characteristics of the source and target modalities. This could lead to a better initialization of model parameters and faster convergence, thereby reducing 
computational demand, improving efficiency, and increasing opportunity for successful deployment.

\section{Ensemble learning}

Data-driven DL models use non-linear methods and learn through stochastic error backpropagation to perform automated feature extraction and classification. These models can only scale up in performance with an increase in the amount of training data and computational resources. Further, their sensitivity to the training data specifics limits their generalization due to learning different sets of weights at each instance of training. This stochastic learning nature results in different predictions referred to as the variance error. Also, there are issues concerning bias errors due to an oversimplified model that results in predictions that are different from the ground truth (GT) thereby placing a higher demand on appropriate threshold selection for obtaining desired performance. Ensemble learning seeks to address these issues by combining predictions of multiple models and resulting in a better performance compared to that of any individual constituent model [11. There are several ensemble approaches such as majority voting, averaging, weighted averaging, stacking, blending, etc.

\section{ROI localization}

Data-driven medical DL models have often been maligned for their "black box" behavior, i.e., inability to make their decision-making process, which is critical for their clinical use, clear. This results in an apparent opaque relationship between input and predictions. This is often due to their massive architectural depth resulting in a large number of model parameters and lack of decomposability into individual explainable components. Further, multiple non-linear processing units perform complex data transformations that can result in unpredictable behavior. This opacity is a serious bottleneck in their use in deriving understandable clinical interpretations.

Variability in the ground truth (GT)

Supervised learning requires a consistent label associated with the appearance of the pathology in the image. However, in medical images, these labels can vary not only for disease stage and shared appearance with other diseases but also with observer expertise and sensitivity to assessment demands. A new pandemic, for example, may bias experts toward higher sensitivity, i.e. they will associate non-specific features with the new disorder because they lack experience with relevant disease manifestation in the image. Therefore, an assessment of observer variability constitutes an essential part of AI-based classification and localization studies. This includes analyzing (i) inter-reader, and (ii) intra-reader variability. It is reported that inter-reader variability tends to be higher than intra-reader variability because multiple observers may have a different opinion on the outlining disease-specific ROI depending on their expertise or personal leanings toward recommending necessary clinical care [12]. Thus, inter-reader variability is a serious bottleneck that may lead to misinterpretation through the "inexact" ROI annotations and also affects supervised learning. Not only can this lead to a false diagnosis or inability to evaluate the true benefit of accurately supplementing clinical-decision making, but it places a greater burden on the number of training images needed to overcome these implicit biases. Thus, it is imperative to conduct inter-reader variability analysis as part of evaluating AI performance. An obvious approach to overcome this challenge might be to compare a collection of annotations by several radiologists using relevant clinical data. However, quantifying expert performance in annotating disease-specific ROI is difficult. This persistent challenge exists because of the difficulty in obtaining or estimating a known true ROI for the task under study. While there exist automated tools to manage inter- and intra-reader variability, these algorithms need to be assessed to warrant their suitability for the task under study. Additionally, it is imperative to determine an appropriate measure for comparing individual expert annotations with each other and with the AI [13]. 
Lack of statistical analysis

Results and methods in a study need to be transparently reported to accurately communicate scientific discovery. Statistical analyses are critical for measuring inherent data variability and their impact on AI performance. They help in evaluating claims and differentiating reasonable and uncertain conclusions. Statistical reporting helps to alleviate issues resulting from incorrect data mining, biased samples, overgeneralization, causality, and violating the assumptions concerning analysis. However, a study of the literature reveals that scientific publications are often limited in presenting statistical analyses of their results 14 .

In this study, we address the aforementioned limitations through a stage-wise systematic approach, as follows: (i) we explore the benefits of repeated CXR-specific pretraining that results in learning CXR modality-specific knowledge, which can be transferred and fine-tuned to improve performance toward COVID-19 detection in CXRs; (ii) we compare the utility of several ImageNet-pretrained CNN models truncated at their empirically determined intermediate layers to that of out-of-the-box ImageNet-pretrained CNNs toward the current task; (iii) we use ensembles of fine-tuned models for COVID-19 detection that are created through various strategies to improve performance compared to any individual constituent model; (iv) we explain learned behavior of individual CNNs and their ensembles using class-selective relevance mapping (CRM)-based localization [15] tools that identify discriminative ROIs involved in detecting COVID-19 viral disease manifestations; (v) we perform ensemble localization to improve localization behavior and compensate for the error due to neglected ROIs by individual CNNs; (vi) we perform exploratory studies to analyze variability in model localization using annotations of two expert radiologists; (vii) we measure statistical significance in performance metrics including Intersection over Union (IoU) and mean average precision (mAP); and, (viii) we perform inter-reader variability analysis using Simultaneous Truth and Performance Level Estimation (STAPLE) 13 using a reference consensus annotation generated from the set of radiologists' annotations. This is compared with individual radiologist annotations and the predicted disease ROI by model ensembles to provide a measure of inter-reader variability and algorithm performance. To our best knowledge, this is the first study to construct ensembles, perform ensemble-based disease ROI localization, and evaluate inter-reader reader variability and algorithm performance toward COVID-19 detection in CXRs.

\section{Related Works}

We describe related works for various topics discussed in this study below.

\section{Image modality-specific transfer learning}

The authors of [16] demonstrated the benefits of transferring knowledge learned from training on a large-scale selection of CXR images and repurposing them toward tuberculosis (TB) detection. They constructed model ensembles and compared their performance with individual models toward classifying CXRs as showing normal lungs or TB -like manifestations. The authors of [17] proposed CXR modality-specific knowledge transfer by retraining the ImageNet-pretrained CNN models on a large-scale selection of CXRs collected from various institutions. This helped in improving generalization of the learned knowledge that was transferred and fine-tuned to detect TB disease-like manifestations in CXRs. The authors performed ensemble learning using the best-performing CNNs to demonstrate better performance in classifying CXRs as belonging to normal or TB-infected classes. At present, the literature on CXR analysis benefiting from modality-specific knowledge transfer particularly applied to detect COVID-19 viral disease manifestations is limited. This leaves room for progress toward evaluating the efficacy of these methods in improving the performance toward 
The authors of 18 used model ensembles to classify CXRs as showing normal lungs or TB-like radiological manifestations. It was observed that an ensemble of custom $\mathrm{CNN}$ and ImageNet-pretrained models delivered superior classification performance with an AUC of 0.99. The authors of [19] evaluated the efficacy of a stacked model ensemble constructed from hand-crafted features/classifiers and DL models toward TB detection in CXRs. CXRs collected from various institutions were used to improve the generalization of the proposed approach. It was observed that the model ensembles delivered better performance than individual constituent models in all performance metrics. Ensemble learning has been applied to detect cardiomegaly in CXRs [20]. The authors observed that DL model ensembles were $92 \%$ accurate as compared to $76.5 \%$ accuracy obtained with hand-crafted features/classifiers. These results demonstrate the superiority of ensemble learning over the traditional approach of evaluating the performance with stand-alone models. Applied to COVID-19 detection in CXRs, the authors of [5] iteratively pruned the DL models and constructed ensembles to improve performance compared to individual constituent models. To this end, the authors observed that the weighted average of iteratively pruned models demonstrated superior classification performance with a 99.01\% accuracy and AUC of 0.9972. Otherwise, the literature available on applying ensemble learning toward COVID-19 detection in chest radiographs is limited.

ROI localization

Exploratory studies in developing explainable and transparent AI solutions toward clinical decision-making are crucial to developing robust solutions for clinical use.

Literature studies reveal several works interpreting the learned behavior of DL models by highlighting pixels that impact prediction scores, with varying intensities. The authors of 21] used deconvolution methods to modify the gradients that resulted in qualitatively improving ROI localization. The authors of [22] inverted image representations using up-CNN models to provide insights into learned feature representations. The authors of 23 generated class-activation maps (CAM) by mapping the prediction class scores back to the deepest convolutional layer. The authors of 24$]$ generalized the use of CAM tools and proposed gradient-weighted CAM (Grad-CAM) methods that can be applied to CNNs with varying architecture. The authors of [15] proposed the CRM algorithm to visualize discriminative ROIs in classifying medical image modalities. The authors measured both positive and negative contributions of the feature map spatial elements in the deepest convolutional layer of the trained models toward making class-specific predictions. It was observed that CRM methods delivered superior localization toward classifying medical imaging modalities compared to CAM-based methods. Applied to the task of localizing COVID-19 viral disease manifestations in CXRs and CT scans, the authors of [7] proposed a DL model that learned the underlying feature representations from volumetric CT scans. It was observed that the model showed better performance with an AUC of 0.96 in detecting COVID-19 viral disease patterns and differentiating them from other non-COVID-19 pneumonia-related opacities. They used CAM-based visualization tools to localize the suspicious ROIs toward detecting COVID-19 viral disease manifestations. The authors of 25] proposed a custom DL model and used Grad-CAM tools to explain their predictions toward COVID-19 detection. The model achieved a sensitivity of $83 \%$ in detecting COVID-19 disease patterns in CXRs. The authors of $[6]$ proposed a weakly-labeled data augmentation approach to increase training data size for recognizing COVID-19 viral related pneumonia opacities in CXRs. They used a strategic approach to train various DL models with non-augmented and weakly-labeled augmented training and evaluated their performance. It was observed that the simple 
addition of CXRs showing COVID-19 viral disease manifestations to weakly labeled augmented training data improved performance. This study revealed that COVID-19 viral disease patterns have a uniquely different presentation compared to non-COVID-19 viral pneumonia-related opacities. The authors used Grad-CAM tools to study the behavior of models trained with non-augmented and augmented data toward localizing COVID-19 viral disease manifestations in CXRs. Otherwise, the literature is limited concerning the use of visualization tools toward COVID-19 detection in CXRs.

Observer variability analysis

Applied to CT scans, the authors of [26] analyzed inter- and intra-radiologist variability in detecting abnormal parenchymal lung manifestations on high-resolution CT scans. They used the Kappa statistic to measure the degree of agreement toward these analyses. A clinically acceptable agreement was observed between the radiologists, but the agreement rate declined when the radiologists were not involved in the regular analysis of thoracic CT scans. Another study [27] analyzed COVID-19 disease manifestations in high-resolution CT scans obtained from patients at the North Sichuan Medical College, Nanchong, China. They assessed inter-observer variability by having $\mathrm{CT}$ readers repeat the data analysis at intervals of three days. A comparison of a set of measurements by the same scan reader was used to assess intra-observer variability. They observed the existence of significant variability in inter- and intra-observer analysis, concerning the extent and density of disease spread. Applied to CXR analysis, the authors of 28 performed an observational study among Russian clinicians in analyzing the variability toward interpreting abnormalities in CXRs. The agreement was analyzed in different scales using the Kappa statistic for a set of 50 CXRs, using different scales. It was observed that there existed only a fair agreement in detecting and localizing abnormalities with a Kappa value of 0.380 and 0.448 , respectively. This demonstrated that limited agreement on interpreting abnormalities resulted in sub-optimal population screening. At present, there is no available literature on the analysis of inter- and/or intra-reader variability applied to COVID-19 detection in CXRs.

\section{Statistical analysis}

The authors of 14 conducted a cross-sectional study toward analyzing the quality of statistical reporting in a random selection of publications in the Journal of Physiology and the British Journal of Pharmacology. The study used samples before and after the publication of an editorial, suggesting measures to adopt in reporting data and statistical analyses. The authors observed no evidence of change in reporting these measures after the editorial publication. It is observed that $90-96 \%$ of papers were not reporting statistical significance measures including p-values to identify the specific groups exhibiting these statistically significant differences in performance. Appropriate statistical analyses are included in the current study.

\section{Materials and methods}

\section{Data collection}

This retrospective study uses the following publicly available datasets:

i) Pediatric CXR dataset: The authors of [29] made available a collection of 5,856 pediatric CXRs showing normal lungs $(\mathrm{n}=1,583$ or bacterial $(\mathrm{n}=2,780)$ or viral pneumonia $(\mathrm{n}=1,493)$ disease manifestations. The data were collected from children age 1 to 5 years at the Guangzhou Children's Medical Center, China. The radiological examinations were performed as a part of routine clinical care. The CXR images are made available in JPEG format, and approximately $2000 \times 2000$ pixels resolution with 8-bit depth.

ii) RSNA CXR dataset: The authors of [30 made available a collection of 26,684 frontal CXRs for a Kaggle challenge. The CXRs are grouped into t normal $(\mathrm{n}=8,851)$ 
and abnormal $(\mathrm{n}=17,833)$ classes; the abnormalities include pneumonia or non-pneumonia related opacities. The CXR images are made available in $1024 \times 1024$ 8-bit pixels resolution and DICOM format.

iii) CheXpert CXR dataset: The authors of 31 made available a collection of 191,219 frontal CXRs showing normal lungs $(\mathrm{n}=17,000)$ or other pulmonary abnormalities $(\mathrm{n}=174,219)$. The CXR images are collected from patients at Stanford University Hospital, California, and are labeled for various thoracic disease manifestations by an automated natural language processing (NLP)-based labeler. The labels are extracted from radiological texts and conform to the Fleischner Society glossary of terms for thoracic imaging.

iv) NIH CXR-14 dataset: The authors of 8 released a collection of 112,120 frontal CXRs, collected from 30,805 patients at the NIH Clinical Center, Maryland. The collection includes CXRs, labeled as showing pulmonary abnormalities $(\mathrm{n}=51,708)$ or normal lungs $(\mathrm{n}=60,412)$. The CXRs were screened to remove personally identifiable information (PII) and ensure patient privacy. The CXRs belonging to the abnormal category are labeled for multiple thoracic disease manifestations using the information extracted from radiological reports using an automated NLP-based labeling algorithm.

v) Twitter-COVID-19 CXR dataset: A radiologist from a hospital in Spain made available a collection of $134 \mathrm{CXR}$ s exhibiting COVID-19 viral pneumonia manifestations, on Twitter (https://twitter.com/ChestImaging). The data were collected from SARS-CoV-2 PCR + subjects and are made available at approximately $2000 \times 2000$ pixels resolution in JFIF format.

vi) Montreal-COVID-19 CXR dataset: The authors of 32 manage a GitHub repository that hosts a collection of CXRs and computed tomography (CT) scans of SARS-CoV-2 + and/or suspected patients. The images are pooled from publications and hospitals through collaboration with physicians and other public resources. As of May 20, 2020, the collection includes 226 CXRs showing COVID-19 viral pneumonia manifestations. The authors didn't provide complete metadata, however, the collection includes CXRs of 131 male patients and 64 female patients; the average age for the COVID-19 group is $58.8 \pm 14.9$ years.

\section{Lung ROI Cropping and preprocessing}

Input data characteristics directly impact DL model learning, which is significant in applications that involve disease detection. For example, clinical decision-making could be adversely impacted by learning irrelevant features. In the case of COVID-19 and other pulmonary diseases, it is vital to limit analysis to the lung ROI and train the models toward learning relevant feature representations from within these pulmonary zones. Literature studies reveal that U-Net-based semantic segmentation delivers commendable performance in segmentation tasks using natural and medical imagery [33]. For this study, we are using a custom U-Net with dropout layers to segment the lung ROI from the background. Gaussian dropouts are used in the encoder, as shown in Fig 1 , to reduce overfitting and provide restrictive regularization [34. A dropout ratio of 0.5 is used after empirical pilot evaluations. The segmentation workflow is shown in Fig 2.

[Fig 1 about here.]

[Fig 2 about here.]

The model is trained on CXRs and their associated lung masks made available by the authors of 35]. Sigmoidal activation is used at the deepest convolutional layer to restrict the mask pixels to the range $(0-1)$. The model is optimized to minimize a combination of binary cross-entropy and dice losses. Callbacks are used to store model checkpoints after each epoch. The best model weights are used for lung mask generation. The model is trained to generate lung masks at $256 \times 256$ pixel resolution 
for various datasets used in this study. The lung boundaries are delineated using the generated masks and are cropped to a bounding box containing the lung pixels. The lung bounding boxes are resized to $256 \times 256$ pixel dimensions and used for further analysis. The cropped lung bounding boxes are further preprocessed as follows: (i) Images are normalized so that the pixel values are restricted to the range $(0-1)$. (ii) Images are passed through a median filter to perform noise removal and edge preservation. (iii) Image pixels are centered through mean subtraction and are standardized to reduce computational complexity.

\section{Repeated CXR-specific pretraining}

Fig 3 illustrates the workflow showing various stages of model training and evaluation.

[Fig 3 about here.]

First, the images are preprocessed to remove irrelevant features by cropping the lung ROI. The cropped images are used for model training and evaluation. We perform repeated CXR-specific pretraining in transferring modality-specific knowledge that is fine-tuned toward detecting COVID-19 viral manifestations in CXRs. Training proceeds in a series of steps. First, the CNNs are trained on a large collection of CXRs to separate normals from those showing abnormalities of any type. Next, we retrain the models from the previous step, focusing on separating CXRs showing bacterial pneumonia or non-COVID pneumonia from normals. Next, we fine-tune the models from the previous step toward the specific separation of CXRs showing COVID-19 pneumonia from normals. Finally, the learned features from these phases of training become parts of the ensembles developed to optimize the detection of COVID-19 pneumonitis from CXRs.

The details of this step-wise approach are discussed as follows. In the first stage of pretraining, a custom CNN and selected ImageNet-pretrained CNN models are retrained on a large selection of CXRs with sufficient diversity due to sourcing from different collections, to coarsely learn the characteristics of normal and abnormal lungs. This CXR-specific pretraining helps in converting the weight layers, specific to the CXRs, in subsequent steps. Table 1 shows the distribution of data used in the first stage of repeated CXR-specific pretraining.

Table 1. Data distribution for the first stage of repeated CXR-specific pretraining. A custom CNN and a selection of ImageNet-pretrained CNNs are retrained on a large selection of CXRs to learn CXR-specific features to categorize them as showing normal or abnormal lungs.

\begin{tabular}{|l|l|l|}
\hline Dataset & Normal & Abnormal \\
RSNA & 8331 & 17833 \\
CheXpert & 16480 & 17000 \\
NIH & 59892 & 51708 \\
Total & 84703 & 86541 \\
\hline
\end{tabular}

The motivation behind this approach is to perform a knowledge transfer from the natural image domain to CXR-domain and learn the characteristics of normal lungs and a wide selection of CXR-specific pulmonary disease manifestations. During this training step, the datasets are split at the patient-level into $90 \%$ for training and $10 \%$ for testing. We randomly allocated $10 \%$ of the training data for validation.

During the second stage of repeated CXR-specific pretraining, the learned knowledge from the first stage pretrained models is transferred and repurposed to classify CXRs as exhibiting normal lungs, bacterial pneumonia, or non-COVID-19 viral pneumonia manifestations. This pretraining is motivated by the biological similarity in non-COVID-19 viral and COVID-19 viral pneumonia. However, there exist distinct 
Table 2. Data distribution for the second stage of repeated CXR-specific pretraining. The first-stage pretrained models are retrained on a collection of CXRs to categorize them as showing normal lungs, bacterial pneumonia, or non-COVID-19 viral pneumonia manifestations. Note that the pediatric CXR dataset predates the onset of the SARS-CoV2 virus, and therefore the viral pneumonia is of non-COVID-19 type.

radiological manifestations between each other as well as with non-viral pneumonia-related opacities [6] 29]. The motivation is to transfer the learned knowledge and fine-tune for COVID-19 detection. Table 2 shows the datasets used and their distribution for this pretraining stage. For the normal class, we pooled CXRs from various collections to introduce generalization and improve model performance. During this pretraining stage, again, the datasets are split at the patient-level into $90 \%$ for training and $10 \%$ for testing. For validation, we randomly allocated $10 \%$ of the training data.

\begin{tabular}{|l|l|l|l|}
\hline Dataset & Normal & Bacterial pneumonia & Non-COVID-19-viral pneumonia \\
CheXpert & 400 & - & - \\
NIH & 400 & - & - \\
Pediatric CXR & 1583 & 2780 & 1493 \\
RSNA & 400 & - & - \\
Total & 2783 & 2780 & 1493 \\
\hline
\end{tabular}

Fine-tuning for COVID-19 detection

The learned knowledge from the second stage of pretraining is transferred and fine-tuned to improve performance in classifying CXRs as showing normal lungs or COVID-19 viral pneumonia disease manifestations. Table 3 shows the datasets used and their distribution toward this fine-tuning stage. We compare this performance to that without repeated CXR-specific pretraining, referred to as Baseline, where the ImageNet-pretrained CNNs are retrained out-of-the-box to categorize the CXRs as showing normal lungs or COVID-19 viral disease manifestations. For the normal class, we pooled CXRs in a patient-specific manner from various collections to introduce generalization and improve model performance. During this training step, we performed a patient-level split of the train and test data as follows: The CXRs from the Montreal-COVID-19 and Twitter-COVID-19 collections are combined $(\mathrm{n}=360)$ where $\mathrm{n}$ is the total number of images in the collection. The data is split at the patient-level into $80 \%$ for training and $20 \%$ for testing. We randomly allocated $10 \%$ of the training data for validation. The test set includes $72 \mathrm{CXRs}$, containing $36 \mathrm{CXRs}$ each from the Montreal-COVID-19 and Twitter-COVID-19 collections.

The GT disease annotations for this test data are set by the verification of publicly identified cases from two expert radiologists, referred to as Rad-1 and Rad-2 hereafter, with a combined experience of 60 years. The radiologists used the web-based VGG Image Annotator tool $\sqrt{36}$ to independently annotate the COVID-19 viral disease-specific ROI in the test collection. The radiologists were shown the chest radiographs in Portable Network Graphics format with a spatial resolution of $1024 \times$ 1024 pixels and were asked to annotate COVID-19 viral disease-specific ROI in the given test set.

Data augmentation

It is well known that large amounts of high-quality data are imperative for DL model training and achieving superior performance. A challenge in the medical image-based DL is the lack of sufficient data. Many studies limit their work to data sourced from a single site. Using limited, single-site data toward model training may result in loss of generalizability and degrade model performance when evaluated on unseen data from other institutions or diverse imaging practices. Under these 
medRxiv preprint doi: https://doi.org/10.1101/2020.07.15.20154385; this version posted July 16, 2020. The copyright holder for this preprint

(which was not certified by peer review) is the author/funder, who has granted medRxiv a license to display the preprint in perpetuity.

It is made available under a CC-BY-NC-ND 4.0 International license .

Table 3. Data distribution for COVID-19 detection. The second-stage pretrained models are fine-tuned to classify CXRs into showing normal lungs or COVID-19 viral patterns.

\begin{tabular}{|l|l|l|}
\hline Dataset & COVID-19+ & Normal \\
CheXpert & - & 120 \\
Montreal-COVID-19 & 226 & - \\
NIH & - & 120 \\
RSNA & - & 120 \\
Twitter-COVID-19 & 134 & - \\
Total & 360 & 360 \\
\hline
\end{tabular}

circumstances, generalizability and performance could be improved by increasing the variability of training data. In this study, we use a diversified data distribution from multiple CXR collections to enhance model generalization and performance in repeated CXR-specific pretraining and fine-tuning stages. Class weights are used to reward the minority classes to prevent biasing error and reduce overfitting. During model training, data are augmented with random horizontal and vertical pixel shifts in the range $(-5-$ to $5)$ and rotations in the degree range (-9 - to 9$)$.

\section{Models}

The following CNN-based DL models are trained and evaluated at various stages of learning performed in this study: (i) a custom wide-residual network (WRN) [37] with dropout, (ii) ResNet-18 [38], (iii) VGG-16 [39], (iv) VGG-19 [39], (v) Xception [40], (vi) Inception-V3 [4], (vii) DenseNet-121 [42, (viii) MobileNet-V2 [43], (ix)

NasNet-Mobile [44]. The models are selected with an idea of increasing the architectural diversity, thereby increasing the representation power, when used in ensemble learning. All computation is done on a Windows ${ }^{\circledR}$ system with Intel Xeon CPU E3-1275 v6 3.80 GHz processor and NVIDIA GeForce ${ }^{\circledR}$ GTX 1050 Ti. We used Keras DL framework with Tensorflow backend, CUDA, and CUDNN libraries to accelerate GPU performance.

Residual CNNs having depths of hundreds of layers suffer from diminishing feature reuse [37. This occurs due to issues with gradient flow, which results in only a few residual blocks learning useful feature representations. A WRN model combats diminishing feature reuse issues by reducing the number of layers and increasing model width [37. The resultant networks are found to exhibit shorter training times with similar or improved accuracy. In this study, we use a custom WRN with dropout regularization. Dropouts provide restrictive regularization, address overfitting issues, and enhance generalization. After empirical observations, we used $5 \times 5$ kernels for the convolutional layers, assigned a dropout ratio of 0.3 , a depth of 16 , and a width of 4 , for the custom WRN used in this study. The resultant architecture is referred further to as custom WRN. Fig 4 shows a WRN block with dropout used in this study. The output from the deepest residual block is average pooled, flattened, and appended to a final dense layer with Softmax activation to predict class probabilities.

[Fig 4 about here.]

As mentioned before, ImageNet-pretrained CNNs have been developed for computer vision tasks with natural images. These models have varying depth and learn diversified feature representations. For medical images that are often available in limited quantities, deeper models may not be optimal and can lead to overfitting and loss of generalization. During the first stage of pretraining, the CNNs are instantiated with 

with (i) zero-padding, (i) a $3 \times 3$ convolutional layer with 1024 feature maps, (ii) a global average pooling (GAP) layer, (iii) a dropout layer with an empirically determined dropout ratio of 0.5 , and (iv) a final dense layer with Softmax activation to output prediction probabilities. These customized models learn CXR-specific feature representations to classify CXR images into normal and abnormal. The custom WRN is initialized with random weights. Fig 5 shows the architecture of the pretrained CNNs used during the first stage of CXR-specific pretraining.

[Fig 5 about here.]

In the second stage, pretrained models from the first stage are truncated at their deepest convolutional layer and appended with (i) GAP layer, (ii) dropout layer (ratio $=0.5$ ), and (iii) dense layer with Softmax activation to output class probabilities for normal, bacterial pneumonia, and non-COVID-19 viral pneumonia. Fig 6 shows the architecture of the customized models used during the second stage of pretraining.

[Fig 6 about here.]

Next, the second-stage pretrained models are truncated at their deepest convolutional layer and appended with (i) GAP layer, (ii) dropout layer (ratio $=0.5$ ), and (iii) dense layer with Softmax activation. The resultant models are fine-tuned to classify the CXRs as belonging to COVID-19+ or normal classes where ' + ' symbolizes COVID-19-positive cases. Fig 7 shows the architecture of the models used toward COVID-19 detection. The models in various learning stages are trained and evaluated using stochastic gradient descent optimization to estimate learning error and classification performance. We used callbacks to check the internal states of the models and store model checkpoints. The model weights delivering superior performance with the test data are used for further analysis.

[Fig 7 about here.]

The performance of the models at various learning stages is evaluated using the following metrics: (i) Accuracy; (ii) Area under curve (AUC); (iii) Sensitivity; (iv) Specificity; (v) Precision; (vi) $\mathrm{F}_{1}$ score; (vii) Matthews correlation coefficient (MCC); (viii) Kappa statistic; and (ix) Diagnostic Odds Ratio (DOR).

\section{Ensemble Learning}

The following ensemble strategies are applied to the fine-tuned models for COVID-19 detection to improve performance: (i) Majority voting; (ii) Simple averaging; and (iii) Weighted averaging. In majority voting, the predictions with maximum votes are considered as final predictions. The average of the individual model predictions is considered the final prediction in a simple averaging ensemble. For a weighted ensemble, we optimized the weights for the model predictions that minimized the total logarithmic loss. This loss decreases as the prediction probabilities converge to true labels. We used the Sequential Least Squares Programming (SLSQP) algorithmic method [45] to perform several iterations of constrained logarithmic loss minimization to converge to the optimal weights for the model predictions.

\section{Inter-reader variability analysis}

Fig 8 shows examples of COVID-19 viral disease-specific ROI annotations on CXRs made by Rad-1 and Rad-2. In this study, we used the well-known STAPLE algorithm to arrive at a consensus reference annotation and use it to evaluate the performance of the top- $\mathrm{N}$ ensembles and to simultaneously assess the performance against each radiologist. 
STAPLE methods are widely used in validating image segmentation algorithms and comparing the performance of experts. Segmentation solutions are treated as a response to a pixel-wise classification problem. The algorithm uses an expectation-maximization (EM) approach that computes a probabilistic estimate of a reference segmented image computed from a collection of expert annotations and weighing them by an estimated level of performance for each expert. It incorporates this knowledge to spatially distribute the segmented structures while satisfying homogeneity constraints. The algorithm is summarized as follows: Let $\mathrm{Q}=\left(\mathrm{q}_{1}, \mathrm{q}_{2}, \ldots, \mathrm{q}_{n}\right)^{N}$ and $\mathrm{R}=\left(\mathrm{r}_{1}, \mathrm{r}_{2}, \ldots\right.$, $\left.\mathrm{r}_{n}\right)^{N}$ denote two column vectors, each containing A elements. The elements in $\mathrm{Q}$ and $\mathrm{R}$ represent sensitivity and specificity parameters, respectively, characterizing one of $\mathrm{N}$ segmentations. Let $\mathrm{D}$ denote an $\mathrm{M} \times \mathrm{N}$ matrix that describes segmentation decisions made for each image pixel. Let $\mathrm{N}$ denote an indicator vector containing $\mathrm{M}$ elements representing hidden, true binary segmentation values. The complete data can be written as $(\mathrm{D}, \mathrm{N})$ and the probability mass function as $\mathrm{f}(\mathrm{D}, \mathrm{N} \mid \mathrm{q}, \mathrm{r})$. The performance level of the experts, characterized by a tuple ( $\mathrm{q}, \mathrm{r})$ is estimated by the EM algorithm, which maximizes $\left(q^{\prime}, r^{\prime}\right)$, the data log-likelihood function, given by,

$$
\left(q^{\prime}, r^{\prime}\right)=\operatorname{argmax}_{q, r} \ln (f(D, N \mid q, r))
$$

We used the following measures including Kappa statistic, sensitivity, specificity, positive predictive value (PPV), and negative predictive value (NPV) to analyze inter-reader variability and assess program performance. We used the STAPLE-generated consensus ROI as to the standard reference and measured its agreement with that generated by the top- $\mathrm{N}$ ensembles and the annotations of Rad-1 and Rad-2. We propose an algorithm to determine the set of True Positive (TP), False Positive (FP), True Negative (TN), and False Negative (FN) for different IoU

thresholds in the range $(0.1-0.7)$. The IoU evaluation metric, also called the Jaccard Index, is widely used in object detection, given by a ratio as shown below:

$$
\text { IoU }(\text { Jaccard Index })=\frac{\text { Area of overlap }}{\text { Area of union }}
$$

where Area of overlap measures the overlap between ROI annotations and Area of union denotes their total combined area. An annotated ROI provided by a given radiologist or that predicted by the top- $\mathrm{N}$ ensemble is considered as a TP if the IoU with the STAPLE-generated consensus ROI is greater than or equal to a given IoU threshold. Each radiologist or top- $\mathrm{N}$ ensemble predicted ROI that produces an IoU less than the threshold or falls outside the consensus ROIs is counted as FP. The FN is defined as a radiologist $\mathrm{ROI}$ or that predicted by the top- $\mathrm{N}$ ensemble that is completely missing when there is an ROI in the consensus ROI. If there is an image with no ROIs on both the masks under test, then we consider it as TN. The values are determined at ROI-level per image and summed to calculate the Kappa statistic given by,

$$
\text { Kappa }=1-\frac{1-p_{o}}{1-p_{e}}
$$

where $\mathrm{p}_{o}$ is the measure of relative observed agreement and $\mathrm{p}_{e}$ denotes the agreement through the hypothetical probability of chance. The values of $\mathrm{p}_{o}$ and $\mathrm{p}_{e}$ are computed as follows:

$$
\begin{gathered}
p_{o}=\frac{(T P+T N)}{T P+F N+F P+T N} \\
p_{e}=\frac{p_{\_} \text {true }}{p_{\text {false }}}
\end{gathered}
$$




$$
\begin{gathered}
p_{-} \text {true }=\frac{(T P+F N)(F P+T P)}{(T P+F N+F P+T N)^{2}} \\
p_{-} \text {false }=\frac{(F P+T N)(F N+T N)}{(T P+F N+F P+T N)^{2}}
\end{gathered}
$$

The sensitivity, specificity, PPV, and NPV parameters are defined as,

$$
\begin{gathered}
\text { Sensitivity }=\frac{T P}{F N+T P} \\
\text { Specificity }=\frac{T N}{F P+T N} \\
P P V=\frac{T P}{F P+T P} \\
N P V=\frac{T N}{F N+T N}
\end{gathered}
$$

Kappa values of 1 and 0 denote complete agreement and disagreement (other than occurring by chance) among the readers, respectively. The value of Kappa becomes negative if the agreement gets worse than random. The algorithm for measuring inter-reader variability is given in Table 4 , where $\mathrm{m}^{1}, \mathrm{~m}^{2}$, and $\mathrm{m}^{p}$ denote the ROI annotations of Rad-1, Rad-2, and that predicted by the top-N ensemble, respectively.

\section{Disease ROI Localization}

In this study, we use the CRM 15 visualization method to interpret the learned behavior of individual models and their ensembles in localizing COVID-19 viral disease-specific ROI manifestations. CRM has been shown to deliver better localization performance than class-activation mapping (CAM)-based visualization. CRM-based localization considers the fact that a feature map spatial element from the trained model's deepest convolution layer would not only contribute to increasing the prediction score for an expected class but also decreasing the score for other class outputs. This helps in maximizing the gap between the scores for various classes. The process results in highly-discriminative ROI localization since it uses incremental mean-squared error (MSE) measured from the output nodes. We construct an ensemble of CRMs by averaging those generated from various fine-tuned models for COVID-19 detection. The size of CRMs from individual models is up-scaled to the size of the image input through a normalization process. This is because the CRMs vary in size depending on the feature map dimensions from the deepest convolutional layers of the individual models. Based on empirical observations, the CRMs are thresholded to remove mapping scores below $20 \%$ to alleviate noise resulting from low mapping scores when constructing CRM ensembles. The resulting ensemble CRM localization is expected to compensate for the error of missed ROI by individual models and enhance COVID-19 disease ROI localization.

We evaluate the effectiveness of CRM-based ensemble localization through the following steps. First, we use CRM-based ROI localization in interpreting the predictions of individual CNNs and compare against the GT annotations provided by each of the two experts. Next, we select the top-3, top-5, and top-7 performing models, ${ }_{\mathbf{5 4 3}}$ 
medRxiv preprint doi: https://doi.org/10.1101/2020.07.15.20154385; this version posted July 16, 2020. The copyright holder for this preprint (which was not certified by peer review) is the author/funder, who has granted medRxiv a license to display the preprint in perpetuity.

It is made available under a CC-BY-NC-ND 4.0 International license .

Table 4. Algorithm to assess inter-reader variability and program performance.

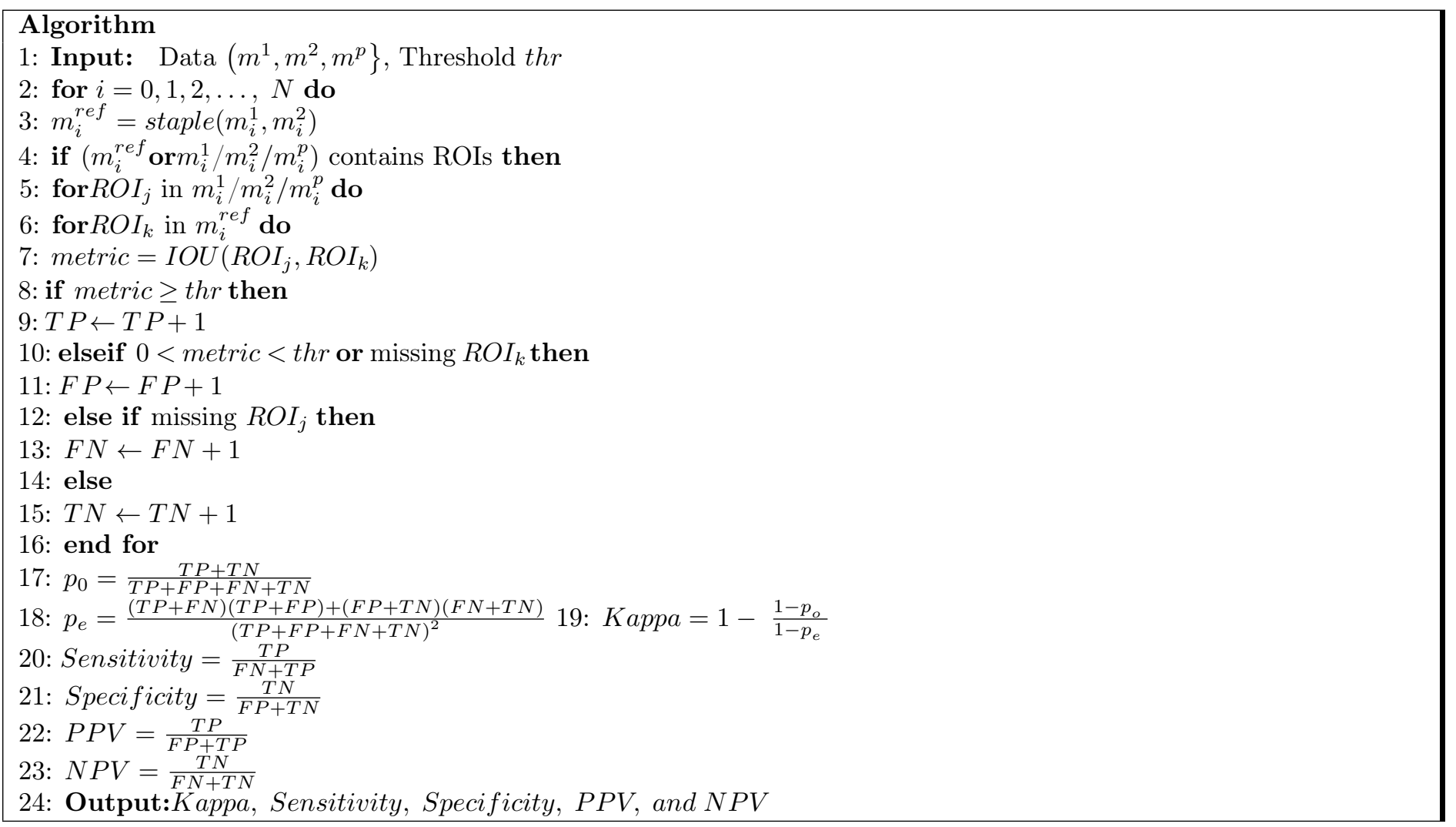

construct ensemble CRMs through an averaging process and compare against each radiologists' independent annotations, and the STAPLE-generated consensus annotation. Finally, we quantitatively compare the ensemble localization performance with each other and against individual CRMs in terms of IoU and mean average precision $(\mathrm{mAP})$ metrics. The mAP score is calculated by taking the mean of average precision (AP) over various IoU thresholds 46 .

Statistical Analysis

Statistical tests are conducted to determine significance in performance differences between the models. We used confidence intervals (CI) to measure model discrimination capability and estimate its precision through the error margin. We measured $95 \% \mathrm{CI}$ as the exact Clopper-Pearson interval for the AUC values obtained by the models in various learning stages. Statistical packages including StatsModels and SciPy are used in these analyses. We performed a one-way analysis of variance (ANOVA) [47] on mAP values obtained with the top- $\mathrm{N}(\mathrm{N}=(3,5,7))$ ensemble to study their localization performance and determine statistical significance among them and against the annotations of each of the radiologist and also the STAPLE-generated consensus annotation. One-way ANOVA tests are performed only if the assumptions of data normality and homogeneity of variances are satisfied for which we performed Shapiro-Wilk and Levene's analyses [47]. Statistical analyses are performed using $\mathrm{R}$ statistical software (Version 3.6.1). 
medRxiv preprint doi: https://doi.org/10.1101/2020.07.15.20154385; this version posted July 16, 2020. The copyright holder for this preprint (which was not certified by peer review) is the author/funder, who has granted medRxiv a license to display the preprint in perpetuity.

It is made available under a CC-BY-NC-ND 4.0 International license .

\section{Results}

Recall that in the first stage of CXR-specific pretraining, we truncated the ImageNet-pretrained CNNs at their intermediate layers to empirically determine the layers that demonstrated superior performance. These empirically determined layers for the various models are shown in Table 5 .

Table 5. Candidate CNN layers delivering superior classification performance during the first stage of CXR-specific pretraining.

\begin{tabular}{|l|l|}
\hline Model & Truncated layers \\
VGG-16 & Block5-conv3 \\
VGG-19 & Block5-conv4 \\
Inception-V3 & Mixed3 \\
Xception & Add_3 \\
DenseNet-121 & Pool3-pool \\
MobileNet-V2 & Block_9_add \\
NASNet-mobile & Activation_94 \\
ResNet-18 & Add_6 \\
\hline
\end{tabular}

The naming conventions for the layers are based on the Keras DL framework. The performance achieved through truncating the models at the selected intermediate layers and appending task-specific heads toward classifying the CXRs is shown in Table 6.

Table 6. Performance metrics achieved during the first-stage of CXR-specific pretraining. The custom WRN is initialized with random weights. Data in parenthesis are $95 \%$ CI for the AUC values measured as the exact Clopper-Pearson interval corresponding to separate 2-sided CI with individual coverage probabilities of $\sqrt{0.95}$. $($ Acc. $=$ Accuracy, $\mathrm{AUC}=$ Area under curve, Sens. $=$ Sensitivity, Spec. $=$ Specificity, Prec. $=$ Precision, $\mathrm{F}_{1}=\mathrm{F}_{1}$ score, $\mathrm{MCC}=$ Matthews correlation coefficient, $\mathrm{DOR}=$ Diagnostics odd ratio). Bold numerical values denote best performances in the respective columns. None of these individual differences are statistically significant.

\begin{tabular}{|l|l|l|l|l|l|l|l|l|l|}
\hline Models & Acc. & AUC (CI) & Sens. & Spec. & Prec. & F 1 & MCC & Kappa & DOR \\
Custom WRN & 0.6696 & $0.722(0.7153,0.7287)$ & 0.6566 & 0.6828 & 0.6763 & 0.6663 & 0.3395 & 0.3393 & 4.12 \\
VGG-16 & 0.6874 & $0.7397(0.7331,0.7463)$ & 0.6641 & 0.711 & 0.6988 & 0.6810 & 0.3755 & 0.3750 & 4.87 \\
VGG-19 & $\mathbf{0 . 6 9 1 3}$ & $\mathbf{0 . 7 4 3 5}(\mathbf{0 . 7 3 7 4}$, & 0.6651 & 0.7178 & 0.704 & 0.6840 & $\mathbf{0 . 3 8 3 3}$ & $\mathbf{0 . 3 8 2 7}$ & $\mathbf{5 . 0 6}$ \\
& & $\mathbf{0 . 7 5 0 6 )}$ & & & & & & & \\
Inception-V3 & 0.6842 & $0.7375(0.7309,0.7441)$ & 0.6186 & 0.7506 & 0.7145 & 0.6631 & 0.3723 & 0.3689 & 4.89 \\
Xception & 0.6727 & $0.7287(0.7220,0.7354)$ & 0.6364 & 0.7094 & 0.6885 & 0.6614 & 0.3466 & 0.3456 & 4.28 \\
DenseNet-121 & 0.6827 & $0.7416(0.7350,0.7482)$ & $\mathbf{0 . 7 5 8 9}$ & 0.606 & 0.6603 & $\mathbf{0 . 7 0 6 2}$ & 0.3692 & 0.3650 & 4.85 \\
NasNet- & 0.6820 & $0.7347(0.7281,0.7413)$ & 0.5802 & $\mathbf{0 . 7 8 4 9}$ & $\mathbf{0 . 7 3 1 3}$ & 0.6471 & 0.3728 & 0.3647 & 5.05 \\
Mobile & & & & & & & & & \\
MobileNet-V2 & 0.6844 & $0.7426(0.7360,0.7492)$ & 0.7007 & 0.668 & 0.6805 & 0.6904 & 0.3688 & 0.3686 & 4.72 \\
ResNet-18 & 0.6821 & $0.7338(0.7272,0.7404)$ & 0.7307 & 0.6332 & 0.6679 & 0.6979 & 0.3657 & 0.3640 & 4.69 \\
\hline
\end{tabular}

From Table 6, we observe that the AUC values are not statistically significantly different across the models $(\mathrm{p}>0.05)$. The DOR provides a measure of diagnostic accuracy and estimation of discriminative power. A high DOR is obtained by a model that exhibits high sensitivity and specificity with low FPs and FNs. Considering AUC and DOR values, VGG-19 demonstrates better performance followed by NasNet-Mobile in classifying CXRs into normal and abnormal categories. A model with higher AUC indicates that it is more capable of distinguishing TNs and TPs. Also considering MCC and Kappa statistic metrics, VGG-19 outperformed other models. The confusion matrix, 
ROC curves, and normalized Sankey flow diagram obtained using the VGG-19 model toward this classification task are shown in Fig 9.

[Fig 9 about here.]

We used a normalized Sankey diagram [48] to visualize model performance. Here, weights are assigned to the classes on the truth (left) and prediction (right) side of the diagram to provide an equal visual representation for the classes on either side. The strips width changes across the plot so that the width of each at the right side represents the fraction of all objects which the model predicts as belonging to a category that truly belongs to each of the categories.

Recall that during the second stage of CXR-specific pretraining, the learned representations from the first-stage pretrained models are transferred and fine-tuned to classify CXRs as showing normal lungs, bacterial proven pneumonia, or non-COVID-19 viral pneumonia. The performance achieved by the second-stage pretrained models is shown in Table 7.

Table 7. Performance metrics achieved by the models during the second stage of CXR-specific pretraining.Bold numerical values denote best performances in the respective columns. None of these individual differences are statistically significant.

\begin{tabular}{|l|l|l|l|l|l|l|l|l|l|}
\hline Models & Acc. & AUC (CI) & Sens. & Spec. & Prec. & F 1 & MCC & Kappa & DOR \\
Custom WRN & 0.7007 & $0.8589(0.8332,0.8846)$ & 0.7007 & 0.8068 & 0.74 & 0.671 & 0.5326 & 0.5136 & 9.78 \\
VGG-16 & 0.8879 & $0.9735(0.9616,0.9854)$ & 0.8879 & 0.9298 & 0.896 & 0.8773 & 0.8312 & 0.8214 & 104.91 \\
VGG-19 & 0.8922 & $0.9739(0.9621,0.9857)$ & 0.8922 & 0.9304 & 0.906 & 0.8825 & 0.8389 & 0.8281 & 110.64 \\
Inception-V3 & 0.9135 & $0.9792(0.9699,0.9895)$ & 0.9135 & 0.9518 & 0.9120 & 0.9110 & 0.8656 & 0.8644 & 180.97 \\
Xception & 0.905 & $0.9714(0.9590,0.9838)$ & 0.905 & 0.943 & 0.9064 & 0.9017 & 0.8532 & 0.8503 & 157.61 \\
DenseNet-121 & $\mathbf{0 . 9 1 7 7}$ & $\mathbf{0 . 9 8 3 5 ( 0 . 9 7 4 0 ,}$ & $\mathbf{0 . 9 1 7 7}$ & $\mathbf{0 . 9 5 1 9}$ & $\mathbf{0 . 9 1 8 7}$ & $\mathbf{0 . 9 1 4 1}$ & $\mathbf{0 . 8 7 3 6}$ & $\mathbf{0 . 8 7 0 4}$ & $\mathbf{2 2 0 . 6 8}$ \\
& & $\mathbf{0 . 9 9 3 0 )}$ & & & & & & & \\
NasNet- & 0.9163 & $0.9819(0.9720,0.9918)$ & 0.9163 & 0.9477 & 0.9222 & 0.9106 & 0.8674 & 0.8674 & 198.38 \\
Mobile & & & & & & & & & \\
MobileNet-V2 & 0.9121 & $0.9812(0.9711,0.9913)$ & 0.9121 & 0.952 & 0.9113 & 0.9098 & 0.8637 & 0.8621 & 205.81 \\
ResNet-18 & 0.8936 & $0.9738(0.9620,0.9856)$ & 0.8936 & 0.9329 & 0.8997 & 0.8849 & 0.8383 & 0.8309 & 116.77 \\
\hline
\end{tabular}

We observed no statistically significant difference in AUC values achieved with the models during this pretraining stage $(\mathrm{p}>0.05)$. Considering DOR, DenseNet-121 demonstrated better performance (220.68) followed by MobileNet-V2 (205.81) in categorizing the CXRs as showing normal lungs, bacterial pneumonia, or non-COVID-19 viral pneumonia. Considering $\mathrm{MCC}$ and $\mathrm{F}_{1}$ score metrics that consider sensitivity and precision to determine model generalization, DenseNet-121 outperformed other models. The confusion matrix, ROC curves, and normalized Sankey flow diagram obtained using the DenseNet-121 model toward this classification task are shown in Fig 10.

[Fig 10 about here.]

The second stage pretrained models are truncated at their deepest convolutional layer, appended with task-specific heads, and fine-tuned to classify the CXRs as belonging to COVID-19+ or normal categories. Table 8 shows the performance metrics achieved by the models toward this task.

We observed no statistically significant difference in AUC values $(\mathrm{p}>0.05)$ achieved by the fine-tuned models. Considering DOR, ResNet-18 demonstrated better performance (83.2) followed by DenseNet-121 (51.54) in categorizing the CXRs as showing normal lungs or manifesting COVID-19 viral disease. The custom WRN, 
medRxiv preprint doi: https://doi.org/10.1101/2020.07.15.20154385; this version posted July 16, 2020. The copyright holder for this preprint (which was not certified by peer review) is the author/funder, who has granted medRxiv a license to display the preprint in perpetuity.

It is made available under a CC-BY-NC-ND 4.0 International license .

Table 8. Performance metrics achieved with fine-tuning the second-stage pretrained models for COVID-19 detection.Bold numerical values denote best performances in the respective columns. Overall, ResNet-18 showed the best performance but individual metrics are not statistically different from other models.

\begin{tabular}{|l|l|l|l|l|l|l|l|l|l|}
\hline Models & Acc. & AUC (CI) & Sens. & Spec. & Prec. & F 1 & MCC & Kappa & DOR \\
D-WRN & 0.8333 & $0.9043(0.8562,0.9524)$ & $\mathbf{0 . 9 0 2 8}$ & 0.7639 & 0.7927 & 0.8442 & 0.6732 & 0.6667 & 30.06 \\
VGG-16 & 0.8681 & $0.9302(0.8885,0.9719)$ & 0.8473 & 0.8889 & 0.8841 & 0.8653 & 0.7368 & 0.7361 & 44.4 \\
VGG-19 & 0.8611 & $0.9176(0.8726,0.9626)$ & 0.9028 & 0.8195 & 0.8334 & 0.8667 & 0.7248 & 0.7222 & 42.17 \\
Inception-V3 & 0.8611 & $0.9123(0.8660,0.9586)$ & $\mathbf{0 . 9 0 2 8}$ & 0.8195 & 0.8334 & 0.8667 & 0.7248 & 0.7222 & 42.17 \\
Xception & 0.8681 & $0.9297(0.8879,0.9715)$ & 0.8334 & 0.9028 & 0.8956 & 0.8634 & 0.7379 & 0.7361 & 46.47 \\
DenseNet-121 & 0.875 & $0.9386(0.8993,0.9779)$ & $\mathbf{0 . 9 0 2 8}$ & 0.8473 & 0.8553 & 0.8784 & 0.7512 & 0.75 & 51.54 \\
NasNet- & 0.8542 & $0.911(0.8644,0.9576)$ & 0.8612 & 0.8473 & 0.8494 & 0.8552 & 0.7085 & 0.7083 & 34.43 \\
Mobile & & & & & & & & & \\
MobileNet-V2 & 0.875 & $0.925(0.8819,0.9681)$ & 0.8473 & 0.9028 & 0.8971 & 0.8715 & 0.7512 & 0.75 & 51.54 \\
ResNet-18 & $\mathbf{0 . 8 9 5 8}$ & $\mathbf{0 . 9 4 9 0 ( 0 . 9 1 3 2 ,}$ & 0.8612 & $\mathbf{0 . 9 3 0 6}$ & $\mathbf{0 . 9 2 5 4}$ & $\mathbf{0 . 8 9 2 1}$ & $\mathbf{0 . 7 9 3 6}$ & $\mathbf{0 . 7 9 1 7}$ & $\mathbf{8 3 . 2}$ \\
& & $\mathbf{0 . 9 8 5 4 )}$ & & & & & & & \\
\hline
\end{tabular}

Inception-V3, and DenseNet-121 are found to be equally sensitive (0.9028) toward this classification task. However, the ResNet-18 fine-tuned model demonstrated better performance with other performance metrics including accuracy, AUC, specificity, precision, $\mathrm{F}_{1}$ score, $\mathrm{MCC}$, and Kappa statistic. The confusion matrix, ROC curves, and normalized Sankey flow diagram obtained using the ResNet-18 model toward this classification task are shown in Fig 11.

[Fig 11 about here.]

Feature embedding visualization

We visualized the deepest convolutional layer feature embedding for the ResNet-18 fine-tuned model, using the t-Distributed Stochastic Neighbor Embedding (t-SNE) algorithm [49]. We used t-SNE to visualize the embedding of the 1024-dimensional feature space into 2 dimensions, as shown in Fig 12. It is observed that the feature space for the normal and COVID-19+ classes is well-separated and clustered to facilitate the classification task.

[Fig 12 about here.]

The performance obtained with the fine-tuned models is compared to the Baseline, as shown in Table 9. The Baseline refers to out-of-the-box ImageNet-pretrained CNNs that are retrained toward this classification task. The custom WRN is initialized with randomized weights for the Baseline task.

As observed in Table 9, the fine-tuned models achieved better performance compared to their baseline counterparts. The AUC metrics achieved with the fine-tuned custom WRN, VGG-16, VGG-19, and NasNet-Mobile models are observed to be statistically significantly different $(\mathrm{p}<0.05)$ compared to their baseline, untuned counterparts. We observed a marked reduction in the number of trainable parameters for the fine-tuned models. The fine-tuned DenseNet-121 model showed a $54.51 \%$ reduction in the number of trainable parameters while delivering better performance as compared to its baseline counterpart. The same holds true for ResNet-18 (46.05\%), Inception-V3 (42.36\%), Xception (37.57\%), MobileNet-V2 (37.38\%), and NasNet-Mobile (11.85\%) with the added benefit of improved performance compared to their baseline models.

ROI visualization

We performed visualization studies to compare how the fine-tuned models and their baseline counterparts localize the ROIs in a CXR manifesting COVID-19 viral patterns. 
medRxiv preprint doi: https://doi.org/10.1101/2020.07.15.20154385; this version posted July 16, 2020. The copyright holder for this preprint (which was not certified by peer review) is the author/funder, who has granted medRxiv a license to display the preprint in perpetuity. It is made available under a CC-BY-NC-ND 4.0 International license .

Table 9. Performance metrics achieved during fine-tuning the second-stage pretrained models for COVID-19 detection is compared with theBaseline. The Baseline refers to retraining out-of-the-box ImageNet-pretrained CNNs toward this task. Bold numerical values show a reduction in the number of parameters.

\begin{tabular}{|c|c|c|c|c|c|c|c|c|c|c|c|}
\hline $\begin{array}{l}\text { Mod- } \\
\text { els }\end{array}$ & Method & Acc. & AUC (CI) & Sens. & Spec. & Prec. & $\mathbf{F}_{1}$ & MCC & Kappa & DOR & $\begin{array}{l}\text { Para. } \\
\text { Reduction (\%) }\end{array}$ \\
\hline $\begin{array}{l}\text { Cus- } \\
\text { tom }\end{array}$ & Baseline & 0.7897 & $\begin{array}{l}0.8014(0.7362 \\
0.8666)\end{array}$ & 0.6742 & 0.8675 & 0.8396 & 0.7478 & 0.5611 & 0.5433 & 14.34 & - \\
\hline WRN & $\begin{array}{l}\text { Fine- } \\
\text { tuned }\end{array}$ & 0.8333 & $\begin{array}{l}0.9043(0.8562, \\
0.9524)\end{array}$ & 0.9028 & 0.7639 & 0.7927 & 0.8442 & 0.6732 & 0.6667 & 30.06 & 0 \\
\hline $\begin{array}{l}\text { VGG- } \\
16\end{array}$ & Baseline & 0.7708 & $\begin{array}{l}0.7993(0.7338, \\
0.8648)\end{array}$ & 0.6667 & 0.875 & 0.8422 & 0.7442 & 0.5539 & 0.5416 & 14.01 & - \\
\hline & $\begin{array}{l}\text { Fine- } \\
\text { tuned }\end{array}$ & 0.8681 & $\begin{array}{l}0.9302(0.8885 \\
0.9719)\end{array}$ & 0.8473 & 0.8889 & 0.8841 & 0.8653 & 0.7368 & 0.7361 & 44.4 & 0 \\
\hline $\begin{array}{l}\text { VGG- } \\
19\end{array}$ & Baseline & 0.7847 & $\begin{array}{l}0.8176(0.7545 \\
0.8807)\end{array}$ & 0.8334 & 0.7362 & 0.7595 & 0.7948 & 0.5722 & 0.5694 & 13.97 & - \\
\hline & $\begin{array}{l}\text { Fine- } \\
\text { tuned }\end{array}$ & 0.8611 & $\begin{array}{l}0.9176(0.8726 \\
0.9626)\end{array}$ & 0.9028 & 0.8195 & 0.8334 & 0.8667 & 0.7248 & 0.7222 & 42.17 & 0 \\
\hline Inceptio & Baseline & 0.8472 & $\begin{array}{l}0.9285(0.8864, \\
0.9706)\end{array}$ & 0.8473 & 0.8473 & 0.8473 & 0.8473 & 0.6945 & 0.6944 & 30.79 & - \\
\hline V3 & $\begin{array}{l}\text { Fine- } \\
\text { tuned }\end{array}$ & 0.8611 & $\begin{array}{l}0.9123(0.8660, \\
0.9586)\end{array}$ & 0.9028 & 0.8195 & 0.8334 & 0.8667 & 0.7248 & 0.7222 & 42.17 & 42.36 \\
\hline Xceptio & Baseline & 0.8472 & $\begin{array}{l}0.9215(0.8775 \\
0.9655)\end{array}$ & 0.9028 & 0.7917 & 0.8125 & 0.8553 & 0.6988 & 0.6944 & 35.31 & - \\
\hline & $\begin{array}{l}\text { Fine- } \\
\text { tuned }\end{array}$ & 0.8681 & $\begin{array}{l}0.9297(0.8879 \\
0.9715)\end{array}$ & 0.8334 & 0.9028 & 0.8956 & 0.8634 & 0.7379 & 0.7361 & 46.47 & 37.57 \\
\hline DenseN & Baseline & 0.8333 & $\begin{array}{l}0.9153(0.8698 \\
0.9608)\end{array}$ & 0.9028 & 0.7639 & 0.7927 & 0.8442 & 0.6732 & 0.6667 & 30.06 & - \\
\hline 121 & $\begin{array}{l}\text { Fine- } \\
\text { tuned }\end{array}$ & 0.8750 & $\begin{array}{l}0.9386(0.8993 \\
0.9779)\end{array}$ & 0.9028 & 0.8473 & 0.8553 & 0.8784 & 0.7512 & 0.75 & 51.54 & 54.51 \\
\hline NasNet- & Baseline & 0.7778 & $\begin{array}{l}0.8502(0.7919, \\
0.9085)\end{array}$ & 0.8473 & 0.7084 & 0.744 & 0.7923 & 0.561 & 0.5556 & 13.48 & - \\
\hline Mobile & $\begin{array}{l}\text { Fine- } \\
\text { tuned }\end{array}$ & 0.8542 & $\begin{array}{l}0.911(0.8644 \\
0.9576)\end{array}$ & 0.8612 & 0.8473 & 0.8494 & 0.8552 & 0.7085 & 0.7083 & 34.43 & 11.85 \\
\hline MobileN & $\begin{array}{l}\text { Baseline } \\
\mathrm{t}-\end{array}$ & 0.8681 & $\begin{array}{l}0.9325(0.8915 \\
0.9735)\end{array}$ & 0.8473 & 0.8889 & 0.8841 & 0.8653 & 0.7368 & 0.7361 & 44.4 & - \\
\hline $\mathrm{V} 2$ & $\begin{array}{l}\text { Fine- } \\
\text { tuned }\end{array}$ & 0.8750 & $\begin{array}{l}0.925(0.8819 \\
0.9681)\end{array}$ & 0.8473 & 0.9028 & 0.8971 & 0.8715 & 0.7512 & 0.75 & 51.54 & 37.38 \\
\hline ResNet- & Baseline & 0.8542 & $\begin{array}{l}0.9302(0.8885 \\
0.9719)\end{array}$ & 0.9167 & 0.7917 & 0.8149 & 0.8628 & 0.714 & 0.7083 & 41.83 & - \\
\hline 18 & $\begin{array}{l}\text { Fine- } \\
\text { tuned }\end{array}$ & 0.8958 & $\begin{array}{l}0.9477(0.9130 \\
0.9850)\end{array}$ & 0.8612 & 0.9306 & 0.9254 & 0.8921 & 0.7936 & 0.7917 & 83.2 & 46.05 \\
\hline
\end{tabular}

Fig 13 shows the following: (i) a CXR with COVID-19 disease consensus ROI obtained with STAPLE using Rad-1 and Rad-2 annotations, and (ii) the ROI localization achieved with various fine-tuned models and their baseline counterparts.

[Fig 13 about here.]

We extracted the features from the deepest convolution layer of the fine-tuned models and their baseline counterparts. We used CRM tools to localize the pixels involved in predicting the CXR images as showing COVID-19 viral disease patterns. As 
observed in Fig. 13, the baseline models demonstrated poor disease ROI localization, compared to the fine-tuned models. We observed that the fine-tuned models learned salient ROI feature representations, matching the experts' knowledge about the disease ROI. The localization excellence of the fine-tuned models can be attributed to (i) CXR-specific knowledge transfer that helped to learn modality-specific characteristics; the learned feature representations are transferred and repurposed for the COVID-19 detection task, and (ii) optimal architecture depth to learn the salient ROI feature representations to classify CXRs to their respective categories. These deductions are supported by poor localization performance of deeper, out-of-the-box ImageNet-pretrained baseline CNNs like DenseNet-121, Inception-V3, and MobileNet-V2, which possibly suffered from overfitting and resulted in poor learning and generalization.

Ensemble studies

We constructed ensembles of the top-3, top-5, and top-7 performing fine-tuned CNNs to evaluate for an improvement in predicting the CXRs as showing normal lungs or COVID-19 viral disease patterns. We used majority voting, simple averaging, and weighted averaging strategies toward this task. In weighted averaging, we optimized the weights for the model predictions to minimize the total logarithmic loss. We used the SLSQP algorithm to iterate through this minimization process and converge to the optimal weights for the model predictions. The results achieved with the various ensemble methods are shown in Table 10.
Table 10. Performance achieved with an ensemble of top-3, top-5, and top-7 fine-tuned models toward COVID-19 detection. Bold numerical values denote best performances in the respective columns. Top- 3 weighted averaging looks best but the AUC differences are not statistically significant.

\begin{tabular}{|c|c|c|c|c|c|c|c|c|c|c|}
\hline Ensemble & Top-N & Acc. & AUC (CI) & Sens. & Spec. & Prec. & $\overline{\mathbf{F}_{1}}$ & MCC & Kappa & DOR \\
\hline \multirow{3}{*}{ Majority voting } & 3 & 0.9028 & 0.9097 (0.8628, & 0.8612 & 0.9167 & 0.9155 & 0.8986 & 0.8084 & 0.8055 & 102.22 \\
\hline & 5 & 0.8819 & $\begin{array}{l}0.9566) \\
0.8819(0.8291 \\
0.9347)\end{array}$ & 0.8612 & 0.9028 & 0.8986 & 0.8795 & 0.7646 & 0.7639 & 57.63 \\
\hline & 7 & 0.8889 & $\begin{array}{l}0.8889(0.8375 \\
0.9403)\end{array}$ & 0.875 & 0.9028 & 0.9000 & 0.8874 & 0.7781 & 0.7778 & 65.02 \\
\hline \multirow{3}{*}{$\begin{array}{l}\text { Simple } \\
\text { averaging }\end{array}$} & 3 & 0.8958 & $\begin{array}{l}0.9483(0.9121 \\
0.9845)\end{array}$ & 0.8889 & 0.9028 & 0.9015 & 0.8952 & 0.7918 & 0.7917 & 74.32 \\
\hline & 5 & 0.8819 & $\begin{array}{l}0.9462(0.9093, \\
0.9831)\end{array}$ & 0.8612 & 0.9028 & 0.8986 & 0.8795 & 0.7646 & 0.7639 & 57.63 \\
\hline & 7 & 0.8819 & $\begin{array}{l}0.9453(0.9081 \\
0.9825)\end{array}$ & 0.875 & 0.8889 & 0.8874 & 0.8812 & 0.764 & 0.7639 & 56.01 \\
\hline \multirow{3}{*}{$\begin{array}{l}\text { Weighted } \\
\text { averaging }\end{array}$} & 3 & 0.9097 & $\begin{array}{l}0.9508(0.9118, \\
0.9844)\end{array}$ & 0.9028 & 0.9445 & 0.9394 & 0.9091 & 0.8196 & 0.8194 & 105.6 \\
\hline & 5 & 0.9028 & $\begin{array}{l}0.9493(0.9134, \\
0.9852)\end{array}$ & 0.875 & 0.9306 & 0.9265 & 0.9000 & 0.8069 & 0.8055 & 93.87 \\
\hline & 7 & 0.8889 & $\begin{array}{l}0.9459(0.9089, \\
0.9829)\end{array}$ & 0.8889 & 0.8889 & 0.8889 & 0.8889 & 0.7778 & 0.7778 & 64.02 \\
\hline
\end{tabular}

We observed no statistically significant difference in the AUC values achieved by the various ensemble methods $(\mathrm{p}>0.05)$. We observed that the performance with top-3 ensembles is better than that of top- 5 and top- 7 ensembles. It is observed that the weighted averaging of top-3 fine-tuned CNNs viz. ResNet-18, MobileNet-V2, and DenseNet-121 demonstrated better performance when their predictions are optimally weighted at $0.6357,0.1428$, and 0.2216 , respectively. This weighted averaging ensemble 
delivered better performance in terms of accuracy, AUC, DOR, Kappa, $\mathrm{F}_{1}$ score, MCC, ${ }_{677}$ and other metrics, as compared to other ensembles. The confusion matrix, ROC curves, 678 and normalized Sankey flow diagram obtained with the weighted averaging of the top-3 fine-tuned CNNs are shown in Fig 14. Table 11 shows the performance achieved in terms of CRM-based IoU and mAP scores by the individual fine-tuned CNNs using the annotations of Rad-1, Rad-2, and STAPLE-generated consensus ROI.

[Fig 14 about here.]

Table 11. Performance achieved in terms of CRM-based IoU and mAP values by the individual fine-tuned CNNs using the radiologists' annotations and STAPLE-generated ROI consensus annotation. Bold numerical values denote best performances in the respective rows.

\begin{tabular}{|c|c|c|c|c|c|c|c|c|c|}
\hline \multirow{3}{*}{$\begin{array}{l}\text { Annota- } \\
\text { tions }\end{array}$} & Parame- & Xcep- & Inception- & DenseNet- & VGG- & VGG- & MobileNet- & ResNet- & NasNet- \\
\hline & ters & tion & V3 & 121 & 19 & & & & Mobile \\
\hline & IOU & 0.0678 & 0.1174 & 0.0799 & 0.0854 & 0.1076 & 0.0644 & 0.0972 & 0.1000 \\
\hline \multirow[t]{3}{*}{ Rad-1 } & $\begin{array}{l}\mathrm{mAP} \\
@[0.1: 0.7]\end{array}$ & 0.0571 & 0.1142 & 0.0697 & 0.0645 & 0.0986 & 0.0712 & 0.0593 & 0.075 \\
\hline & Ranking & 8 & 1 & 5 & 6 & 2 & 4 & 7 & 3 \\
\hline & IOU & 0.2146 & 0.2567 & 0.2398 & 0.2183 & 0.2230 & 0.1825 & 0.2293 & 0.2569 \\
\hline \multirow[t]{3}{*}{ Rad-2 } & $\begin{array}{l}\mathrm{mAP} \\
@[0.1: 0.7]\end{array}$ & 0.146 & 0.206 & 0.1858 & 0.1643 & 0.1882 & 0.1467 & 0.1742 & 0.2186 \\
\hline & Ranking & 8 & 2 & 4 & 6 & 3 & 7 & 5 & 1 \\
\hline & IOU & 0.0670 & 0.1337 & 0.0916 & 0.0951 & 0.1267 & 0.0713 & 0.1126 & 0.1095 \\
\hline \multirow[t]{2}{*}{ STAPLE } & $\mathrm{mAP}$ & 0.0603 & 0.1213 & 0.0792 & 0.073 & 0.1068 & 0.0775 & 0.0648 & 0.0851 \\
\hline & Ranking & 8 & 1 & 4 & 6 & 2 & 5 & 7 & 3 \\
\hline
\end{tabular}

We observed that the model ROI predictions achieved varying IoU and mAP scores with the annotations of Rad-1, Rad-2, and the STAPLE-generated ROI consensus. For Rad-1, the fine-tuned Inception-V3 model demonstrated higher values for the average IoU and mAP metrics. For Rad-2, we observed that the fine-tuned NasNet-Mobile outperformed other models. With STAPLE-generated consensus ROI, the Inception-V3 model outperformed other models in localizing COVID-19 viral disease-specific ROI.

The precision-recall (PR) curves of the best performing models using Rad-1, Rad-2, and the STAPLE-generated consensus ROI are shown in Fig 15. These curves are generated for varying IoU thresholds in the range $(0.1-0.7)$. The confidence score threshold is varied to generate each curve. For a given fine-tuned model, we define the confidence score as the highest heat map value in the predicted ROI weighted by the classification score at the output nodes. We considered the ROI predictions as TP when the IoU and confidence scores are higher than their corresponding thresholds. For a given PR curve, we computed the AP score as the average of the precision across all recall values.

[Fig 15 about here.]

The following are the important observations from this localization study: (i) The accuracy of a model is not related to disease ROI localization. From Table 6, we observed that the fine-tuned ResNet-18 model is highly accurate, followed by DenseNet-121 and MobileNet-V2, in classifying the CXRs as belonging to the COVID-19 viral category. However, while localizing disease-specific ROI, the Inception-V3, VGG-16, and NasNet-Mobile fine-tuned models delivered superior ROI 
localization performance compared to other models. This underscores the fact that the classification accuracy of a model is not an optimal measure to interpret their learned behavior. Localization studies are indispensable to understand the learned features and compare them to the expert knowledge for the problem under study. These studies provide comprehensive qualitative and quantitative measures of the learning capacity of the model and its generalization ability.

Next, we constructed an ensemble of CRMs through averaging the ROI localization for the top-3, top-5, and top-7 fine-tuned models. We ranked the models based on the IoU and mAP scores. The localization performance achieved with the various ensemble CRMs is shown in Table 12.

Table 12. IOU and mAP values obtained by top-3, top-5, and top-7 ensembles using annotations of Rad-1, Rad-2, and STAPLE-generated consensus ROI annotations. Bold numerical values denote best performances in the respective rows.

\begin{tabular}{|l|l|l|l|l|}
\hline Annotations & Parameters & Top-3 & Top-5 & Top-7 \\
Rad-1 & IOU & $\mathbf{0 . 1 3 4 3}$ & 0.0994 & 0.1236 \\
& I mAP@ $[0.1: 0.7]$ & $\mathbf{0 . 1 2 6 4}$ & 0.0767 & 0.0753 \\
Rad-2 & IOU & 0.2673 & $\mathbf{0 . 2 9 5 5}$ & 0.2865 \\
& I mAP@ $[0.1: 0.7]$ & 0.2179 & $\mathbf{0 . 2 3 5 2}$ & 0.2292 \\
STAPLE & IOU & $\mathbf{0 . 1 5 1 8}$ & 0.1193 & 0.1350 \\
& I mAP@ $[0.1: 0.7]$ & $\mathbf{0 . 1 3 5 2}$ & 0.0924 & 0.0916 \\
\hline
\end{tabular}

From Table 12, we observed that the ensemble CRMs delivered superior ROI localization performance compared to that achieved with the individual models. However, the number of models in the top-performing ensembles varied. While using the annotations of Rad-1, we observed that the ensemble of the top-3 models demonstrated higher values for IoU and mAP than other ensembles. However, for Rad-2, the ensemble of the top-5 models demonstrated superior localization with IoU and mAP values of 0.2955 and 0.2352 , respectively. The ensemble of top- 3 fine-tuned models demonstrated higher values for IoU and mAP scores compared to other models while using STAPLE-generated ROI consensus annotation. Considering this study, we observed that averaging the CRMs of more than top- 5 fine-tuned models didn't improve performance but rather it saturates ROI localization. The PR curves obtained with the top-N ensemble CRMs using Rad-1, Rad-2, and STAPLE-generated consensus ROI are shown in Fig 16.

[Fig 16 about here.]

Instances of CXRs showing ROI annotations of Rad-1, Rad-2, top-3 ensemble using STAPLE-generated ROI consensus (referred to as program hereafter), and the STAPLE-generated ROI consensus annotation are shown in Fig 17.

[Fig 17 about here.]

Fig 18 shows the following: (A) an ensemble CRM generated with the top-3 fine-tuned models that delivered superior localization performance using STAPLE-generated ROI consensus annotation, and (B) an ensemble CRM generated with the top-5 fine-tuned models that delivered superior localization performance using the annotations of Rad- 2 .

[Fig 18 about here.]

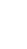


We observe that the CRMs obtained using individual models in the top-N ensemble highlight ROI to varying extents. The ensemble CRM averages the ROIs localized with individual CRMs to highlight the disease-specific ROI involved in class prediction. The ensemble CRMs have a superior IoU value, compared to that of individual CRMs; the ensemble CRM improved localization performance as compared to individual ROI localization. This underscores the fact that ensemble localization improves performance and ability to generalize, conforming to the experts' knowledge about COVID-19 viral disease manifestations.

\section{Statistical Analysis}

To perform a one-way ANOVA analysis, we investigated whether the assumptions of data normality and homogeneous variances are satisfied. We used the Shapiro-Wilk test to investigate for normal distribution of the data and Levene's test, for homogeneity of variances, using mAP scores obtained with the top-N ensembles. We plotted the residuals to investigate if the assumption of normal residual distribution is satisfied. Fig 19 shows the following: (A) The mean plot for the mAP scores obtained by the top-N ensembles using Rad-1, Rad-2, and STAPLE-generated consensus ROI annotations, and (B) a plot of the quantiles of the residuals against that of the normal distribution.

[Fig 19 about here.]

It is observed from Fig 19B that all the points fall approximately along with a 45-degree reference that shows that the assumption of the normal distribution is satisfied. Table 13 shows the consolidated results of Shapiro-Wilk, Levene, and one-way ANOVA analyses.

Table 13. Consolidated results of Shapiro-Wilk, Levene, and one-way ANOVA analyses.

\begin{tabular}{|l|l|l|l|l|}
\hline Metric & Shapiro-Wilk test (p ) & Levene's test (p ) & ANOVA (F) & ANOVA (p ) \\
mAP & 0.1014 & 0.3365 & 1.678 & 0.2060 \\
\hline
\end{tabular}

To compute one-way ANOVA, we measure the variance between group means, the variance within the group, and the group sizes. This information is combined to measure statistical significance from the test statistic $\mathrm{F}$ if it follows an F-distribution. In our study, we have three groups (Rad-1, Rad-2, and STAPLE) of 10 observations each, hence the distribution is mentioned as F $(2,27)$. As observed from Table 13, the $\mathrm{p}$-values obtained with the Shapiro-Wilk test are not significant $(\mathrm{p}>0.05)$ and reveals that the normality assumption is satisfied. The result of Levene's test is not statistically significant $(p>0.05)$. That demonstrates that the variance across the mAP values obtained with the annotations of Rad-1, Rad-2, and STAPLE-generated consensus ROI are not statistically significantly different. Since the conditions of data normality and homogeneity of variances are satisfied, we performed one-way ANOVA to explore the existence of a statistically significant difference in the mAP scores. To this end, we observed no statistically significant difference in the mAP scores obtained with Rad-1, Rad-2, and STAPLE-generated consensus ROI $(\mathrm{F}(2,27)=1.678, \mathrm{p}=0.2060)$. This smaller F-value underscores the fact that the null hypothesis $\left(\mathrm{H}_{0}\right)$-all groups demonstrate equal mAP scores- holds good.

\section{Inter-reader variability analysis and performance assessment}

We used the STAPLE-generated consensus ROI as to the standard reference and measured its agreement with that generated by the program and the radiologists. The consensus ROI is estimated from the set of ROI annotations provided by Rad- 1 and Rad-2. STAPLE assumes that Rad-1 and Rad-2 individually annotated ROIs for the given CXRs so that the quality of annotations are captured. We determined the set of TPs, FPs, TNs, and FNs for 10 different IoU thresholds in the range $(0.1-0.7)$ and 
provided a measure of inter-reader variability and program performance using the following metrics: (i) Kappa statistic; (ii) Sensitivity; (iii) Specificity; (iv) PPV; and (v) NPV. These parameters depend on the relative proportion of the disease-specific ROI. An ROI provided by a radiologist or predicted by the program is considered as a TP if the IoU with the consensus ROI is greater than or equal to a given IoU threshold. Each radiologist or program ROI that produces an IoU less than the threshold or falls outside the consensus ROIs is counted as FP. The FN is defined as a radiologist or program ROI that is completely missing when there is a consensus ROI. If there is an image with no ROIs on both the ROI annotations under test, it is considered as TN. Fig 20 shows the variability in Kappa, sensitivity, specificity, and PPV values observed for the Rad-1, Rad-2, and the program.

[Fig 20 about here.]

The estimated Kappa, sensitivity, specificity, PPV, and NPV values that are averaged over 10 different IoU thresholds in the range $(0.1-0.7)$ are shown in Table 14
Table 14. Performance level assessment and inter-reader variability analysis using STAPLE-generated consensus ROI.Bold numerical values denote the best performances in respective columns.

\begin{tabular}{|l|l|l|l|l|l|}
\hline Annotations & Kappa & Sensitivity & Specificity & PPV & NPV \\
Rad - 1 & $\mathbf{0 . 1 8 0 5}$ & $\mathbf{1 . 0}$ & 0.1384 & $\mathbf{0 . 7 1 4 0}$ & $\mathbf{1 . 0}$ \\
Rad - 2 & 0.0080 & $\mathbf{1 . 0}$ & 0.0121 & 0.2877 & $\mathbf{1 . 0}$ \\
Program & 0.0740 & 0.9037 & $\mathbf{0 . 1 4 6 7}$ & 0.5154 & 0.6 \\
\hline
\end{tabular}

The performance assessment as observed from Table 14 indicated that Rad-1 is more specific than Rad-2. The same holds good for the Kappa and PPV metrics. We observed that NPV is 1 for Rad-1 and Rad-2. This is because the number of FNs $=0$, which signifies that none of the radiologists ROI completely missed when there is an ROI in the STAPLE-generated consensus annotation. However, the NPV achieved with the program is 0.6 which underscores the fact the predicted ROIs missed a marked proportion of ROIs in the STAPLE-generated consensus. This assessment indicated that Rad-1 generated annotations similar to that of STAPLE-generated consensus by demonstrating higher values for Kappa, sensitivity, and PPV as compared to Rad-2. We also observed that the program is performing with higher specificity but with lower sensitivity as compared to Rad-1 and Rad-2. These assessments provided feedback indicating the need for program modifications, parameter tuning, and other measures, to improve its localization performance.

\section{Discussion}

There are several salient observations to be made from the analyses reported above. These include the kind of data used in training, the size and variety of data collections, learning ability of various DL architectures informing their selection, need for customizing the models for improved performance, benefits of ensemble learning, and the imperative for localization to measure conformity to the problem.

We observed that repeated CXR-specific pretraining and fine-tuning resulted in improved performance toward COVID-19 detection as compared to the baseline, out-of-the-box, ImageNet pretrained CNNs. This highlights the need to use task-specific modality training resulting in improved model adaption, convergence, reduced bias, and reduced overfitting. This approach may have helped the DL models differentiate distinct radiological manifestations between COVID viral pneumonia and other
799 
non-viral pneumonia-related opacities. An added benefit is that this approach resulted in reductions in both computations and the number of trainable parameters.

It is well-known that neural networks develop or learn implicit rules to convert input data into features for making decisions. These learned rules are opaque to the user and the decisions are difficult to interpret. However, an interpretable model explaining its predictions related to model accuracy doesn't necessarily guarantee that accurate predictions are for the right reasons. Localization studies help observe if the model has learned salient ROI feature representations that agree with expert annotations. In our study, we demonstrate that CRM visualization tools show superior localization performance in localizing COVID-19 viral disease-specific ROIs, particularly for the fine-tuned models compared to the ImageNet-pretrained CNNs.

Model ensembles further improved qualitative and quantitative performance in COVID-19 detection. Ensemble learning compensated mislabeling in individual models by combining their predictions and reduced prediction variance to the training data. Sensitivity also declined slightly, but this decline was not statistically significant. We observed that the weighted averaging ensemble of the top-3 performing fine-tuned models delivered better performance compared to any individual constituent model. The results demonstrate that the detection task benefits from an ensemble of repeated CXR-specific pretrained and fine-tuned models. Ensemble learning also compensates for localization errors in CRMs and missed ROIs by combining and averaging the individual CRMs. Empirical evaluations show that ensemble localization demonstrated superior IoU and mAP scores and they significantly outperform ROI localization by individual CNN models.

It is difficult to quantify individual radiologists' performance in annotating ROIs in medical images. Not only are they the truth standard, but this "truth" is impacted by inherent biases related to a pandemic event like COVID-19 and their clinical exposure and experience. This complexity is compounded further because CXRs offer lower diagnostic sensitivity than CTs for example. So, a conservative assessment of the CXR is likely to result in smaller and more specific truth annotation ROIs. We used STAPLE to compute a probabilistic estimate of expert ROI annotations for the two expert radiologists who contributed to this study. STAPLE assumes these annotations are conditionally independent. The algorithm discovers and quantifies the bias among the experts when they differ in their opinion of the disease-specific ROI annotation. We use STAPLE-generated annotations as GT to assess the variation for every annotation for each expert, where the DL model is also considered as an expert. We observed that the Kappa values obtained using the STAPLE-generated consensus ROI are in a low range $(0-0.2)$. This is probably because of the small number of experts and their inherent biases in assessing COVID-19 cases. Particularly, we note that Rad-1 was very specific in marking the ROIs, whereas Rad-2 annotated larger regions that sometimes accommodated multiple smaller regions into a single ROI. This led to lower IoU value that in turn affected the Kappa value. The pandemic is an evolving situation and CXR manifestations often exhibit biological similarity to non-COVID-19 viral pneumonia. The CXR is not a definitive diagnostic tool and expert views may differ in referring a candidate patient for further review. It would be helpful to conduct a similar analysis with a larger number of experts on a larger patient population. We remain hopeful that health agencies and medical societies will make such image collections available for future research. As more reliable and widely available COVID testing becomes available, the results of that testing could be used with CXRs as an additional important indicator of GT.

Regarding the limitations of our study: (i) The publicly available COVID-19 data collections used are fairly small and may not encompass a wide range of disease pattern variability. An appropriately annotated large-scale collection of CXRs with COVID-19 
viral disease manifestations is necessary to build confidence in the models, improve their robustness, and generalization. (ii) The study is evaluated with the ROI annotations obtained from two expert radiologists. However, it would help to have more radiologists contribute independently in the annotation process and then arrive at a consensus that could reduce annotation errors. (iii) We used conventional convolutional kernels toward this study, however, future research could propose novel convolutional kernels that reduce feature dimensionality and redundancy and result in improved performance with reduced memory and computational requirements. (iv) Ensemble models require markedly high training time, memory, and computational resources for successful deployment and use. However, recent advancements in storage and computing solutions and cloud technology could lead to improvements in this regard.

\section{Conclusions}

In this study, we have demonstrated that a combination of repeated CXR-specific pretraining, fine-tuning, and ensemble learning helped in (a) transferring CXR-specific learned knowledge that is subsequently fine-tuned to improve COVID-19 detection in CXRs; and (b) improving classification generalization and localization performance by reducing prediction variance. Ensemble-based ROI localization helped in improving localization performance by compensating for the errors in individual constituent models. We also performed inter-reader variability analysis and program performance assessment by comparing them with a STAPLE-based estimated reference. This assessment highlighted the opportunity for improving performance through ensemble modifications, requisite parameter optimization, increased task-specific dataset size, and involving "truth" estimates from a larger number of expert collaborators. We believe that the results proposed are useful for developing robust models for tasks involving medical image classification and disease-specific ROI localization.

\section{Acknowledgment}

This study is supported by the Intramural Research Program (IRP) of the National Library of Medicine (NLM) and the National Institutes of Health (NIH).

\section{References}

1. COVID-2019) situation reports. In: World Health Organization (WHO) Situation Reports. Coronavirus disease. 2020;.

2. Rubin GD, Ryerson CJ, Haramati LB, Sverzellati N, Kanne JP, Raoof S, et al. The Role of Chest Imaging in Patient Management During the COVID-19 Pandemic. Chest. 2020;158(1):106-116.

3. ACR Recommendations for the use of Chest Radiography and Computed Tomography (CT) for Suspected COVID-19 Infection; 2020. Available from: https:// www.acr.org/Advocacy-and-Economics/ACR-Position-Statements/ Recommendations-for-Chest-Radiography-and-CT-for-Suspected-COVID19Infection.

4. Bai HX, Hsieh B, Xiong Z, Halsey K, Choi JW, Tran T. Performance of radiologists in differentiating COVID-19 from viral pneumonia on chest $\mathrm{CT}$. Radiology. 2020;.

5. Rajaraman S, Siegelman J, Alderson PO, Folio LS, Folio LR, Antani SK. Iteratively Pruned Deep Learning Ensembles for COVID-19 Detection in Chest X-rays. IEEE Access. 2020;8:1-1. 
6. Rajaraman S, Antani S. Weakly Labeled Data Augmentation for Deep Learning: A Study on COVID-19 Detection in Chest X-Rays. Diagnostics. 2020;10(6):358-358.

7. Li L, Qin L, Xu Z, Yin Y, Wang X, Kong B. Artificial Intelligence Distinguishes COVID-19 from Community Acquired Pneumonia on Chest CT. Radiology. 2020;.

8. Wang X, Peng Y, Lu L, Lu Z, Bagheri M, Summers RM. ChestX-ray8: Hospitalscale Chest X-ray Database and Benchmarks on Weakly-Supervised Classification and Localization of Common Thorax Diseases. Proceedings of the International Conference on Computer Vision (ICCV. 2017; p. 3462-3471.

9. Deng J, Dong W, Socher R, Li L, Li K, Li FF, et al. A large-scale hierarchical image database. Proceedings of the IEEE Conference on Computer Vision and Pattern Recognition. 2009; p. 248-255.

10. Shen D, Wu G, Suk HI. Deep Learning in Medical Image Analysis. Annual Review of Biomedical Engineering. 2017;19(1):221-248.

11. Chowdhury AK, Tjondronegoro D, Chandran V, Trost SG. Ensemble Methods for Classification of Physical Activities from Wrist Accelerometry. Medicine \& Science in Sports \& Exercise. 2017;49(9):1965-1973.

12. Zhao B, Tan Y, Bell DJ, Marley SE, Guo P, Mann H, et al. Exploring intra- and inter-reader variability in uni-dimensional, bi-dimensional, and volumetric measurements of solid tumors on $\mathrm{CT}$ scans reconstructed at different slice intervals. European Journal of Radiology. 2013;82(6):959-968.

13. Warfield SK, Zou KH, Wells WM. Simultaneous Truth and Performance Level Estimation (STAPLE): An Algorithm for the Validation of Image Segmentation. IEEE Transactions on Medical Imaging. 2004;23(7):903-921.

14. Diong J, Butler AA, Gandevia SC, Héroux ME. Poor statistical reporting, inadequate data presentation and spin persist despite editorial advice. PLOS ONE. 2018;13(8):e0202121.

15. Kim I, Rajaraman S, Antani S. Visual Interpretation of Convolutional Neural Network Predictions in Classifying Medical Image Modalities. Diagnostics. $2019 ; 9(2): 38-38$.

16. Yadav O, Passi K, Jain CK. Using Deep Learning to Classify X-ray Images of Potential Tuberculosis Patients. Proceedings of the IEEE International Conference on Bioinformatics and Biomedicine (BIBM). 2018; p. 2368-2375.

17. Rajaraman S, Antani SK. Modality-Specific Deep Learning Model Ensembles Toward Improving TB Detection in Chest Radiographs. IEEE Access. 2020;8:27318-27326.

18. Lakhani P, Sundaram B. Deep Learning at Chest Radiography: Automated Classification of Pulmonary Tuberculosis by Using Convolutional Neural Networks. Radiology. 2017;284(2):574-582.

19. Rajaraman S, Sornapudi S, Kohli M, Antani S. Assessment of an ensemble of machine learning models toward abnormality detection in chest radiographs. Conf Proc IEEE Eng Med Biol Soc. 2019;2019:3689-3692.

20. Islam MT, Aowal MA, Minhaz AT, Islam KA; 2017.Abnormality Detection and Localization in Chest X-Rays using Deep Convolutional Neural Networks. arXiv preprint arXiv: 170509850. 
21. Zeiler MD, Fergus R; 2013.Visualizing and Understanding Convolutional Networks. arXiv preprint arXiv:13112901.

22. Dosovitskiy A, Brox T. Inverting Visual Representations with Convolutional Networks. Proceedings of the IEEE Conference on Computer Vision and Pattern Recognition (CVPR). 2016; p. 4829-4837.

23. Zhou B, Khosla A, Lapedriza A, Oliva A, Torralba A. Learning Deep Features for Discriminative Localization. Proceedings of the IEEE Conference on Computer Vision and Pattern Recognition (CVPR). 2016; p. 2921-2929.

24. Selvaraju RR, Cogswell M, Das A, Vedantam R, Parikh D, Batra D, et al. Visual explanations from deep networks via gradient-based localization. Proceedings of the International Conference on Computer Vision (ICCV. 2017; p. 618-626.

25. Karim MR, Döhmen T, Rebholz-Schuhmann D, Decker S, Cochez M, Beyan O, et al; 2020. DeepCOVIDExplainer: Explainable COVID-19 Predictions Based on Chest Xray Images. arXiv preprint arXiv:200404582.

26. Al-Khawari H, Athyal RP, Al-Saeed O, Sada PN, Al-Muthairi S, Al-Awadhi A. Inter- and intraobserver variation between radiologists in the detection of abnormal parenchymal lung changes on high-resolution computed tomography. Annals of Saudi Medicine. 2010;30(2):129-133.

27. Jiang Y, Guo D, Li C, Chen T, Li R. High-resolution CT features of the COVID-19 infection in Nanchong City: Initial and follow-up changes among different clinical types. Radiology of Infectious Diseases. 2020;

28. Balabanova Y, Coker R, Fedorin I, Zakharova S, Plavinskij S, Krukov N, et al. Variability in interpretation of chest radiographs among Russian clinicians and implications for screening programmes: observational study. BMJ. 2005;331(7513):379-382.

29. Kermany DS, Goldbaum M, Cai W, Valentim C, Liang H, Baxter SL. Identifying Medical Diagnoses and Treatable Diseases by Image-Based Deep Learning. Cell. 2018;172(5):1122-1131.

30. Shih G, Wu CC, Halabi SS, Kohli MD, Prevedello LM, Cook TS. Augmenting the National Institutes of Health Chest Radiograph Dataset with Expert Annotations of Possible Pneumonia. Radiol Artif Intell. 2019;1(1):180041-180041.

31. Irvin J, Rajpurkar P, Ko M, Yu Y, Silviana CI, Chute C. CheXpert: a large chest radiograph dataset with uncertainty labels and expert comparison. Proceedings of the 33rd AAAI conference on artificial intelligence (AAAI). 2019; p. 590-597.

32. Cohen JP, Morrison P, Dao L;. 2020. COVID-19 image data collection. arXiv preprint arXiv:200311597.

33. Hesamian MH, Jia W, He X, Kennedy P. Deep Learning Techniques for Medical Image Segmentation: Achievements and Challenges. Journal of Digital Imaging. 2019;32(4):582-596.

34. Baldi P, Sadowski P. The dropout learning algorithm. Artificial Intelligence. 2014;210:78-122.

35. Candemir S, Antani S. A review on lung boundary detection in chest X-rays. International Journal of Computer Assisted Radiology and Surgery. 2019;14(4):563-576.

36. Dutta A, Zisserman A. The VIA Annotation Software for Images, Audio and Video. Proceedings of the 27th ACM International Conference on Multimedia (MM). 2019; p. 2276-2279. 
37. Zerhouni E, Lanyi D, Viana MP, Gabrani M. Wide residual networks for mitosis detection. Proceedings of the IEEE International Symposium on Biomedical Imaging. 2017; p. 924-928.

38. Zhang HX, Ren S, Sun J. Deep residual learning for image recognition. Proceedings of the International Conference on Computer Vision (ICCV). 2016; p. $770-778$.

39. Simonyan K, Zisserman A. Very deep convolutional networks for large-scale image recognition. Proceedings of the International Conference on Learning Representations (ICLR). 2015; p. 1-14.

40. Chollet F. Xception: Deep learning with depthwise separable convolutions. Proceedings of the IEEE Conference on Computer Vision and Pattern Recognition. 2017; p. 1251-1258.

41. Szegedy C, Vanhoucke V, Ioffe S, Shlens J, Wojna Z. Rethinking the Inception architecture for computer vision. Proceedings of the IEEE Conference on Computer Vision and Pattern Recognition (CVPR). 2016; p. 2818-2826.

42. Liu HZ, Maaten LVD, Weinberger KQ. Densely connected convolutional networks. Proceedings of the International Conference on Computer Vision (ICCV. 2017; p. 4700-4708.

43. Sandler M, Howard AG, Zhu M, Zhmoginov A, Chen LC. MobileNetV2: Inverted Residuals and Linear Bottlenecks. Proceedings of the IEEE Conference on Computer Vision and Pattern Recognition (CVPR). 2018; p. 4510-4520.

44. Pham MY, Zoph GB, Le QV, Dean J. Efficient neural architecture search via parameter sharing. Proceedings of the International Conference on Machine Learning (ICML). 2018; p. 4092-4101.

45. Zahery M, Maes HH, Neale MC. CSOLNP: Numerical Optimization Engine for Solving Non-linearly Constrained Problems. Twin Research and Human Genetics. 2017;20(4):290-297.

46. Lin TY, Maire M, Belongie S, Hays J, Perona P, Ramanan D, et al. Microsoft COCO: common objects in context. Proceedings of the European Conference on Computer Vision (ECCV). 2014; p. 740-755.

47. Kao LS, Green CE. Analysis of Variance: Is There a Difference in Means and What Does It Mean? Journal of Surgical Research. 2008;144(1):158-170.

48. Platzer A, Polzin J, Rembart K, Han PP, Rauer D, Nussbaumer T. BioSankey: Visualization of Microbial Communities Over Time. Journal of Integrative Bioinformatics. 2018;15(4).

49. Acuff NV, Linden J. Using Visualization of t-Distributed Stochastic Neighbor Embedding To Identify Immune Cell Subsets in Mouse Tumors. The Journal of Immunology. 2017;198(11):4539-4546. 


\section{List of Figures}

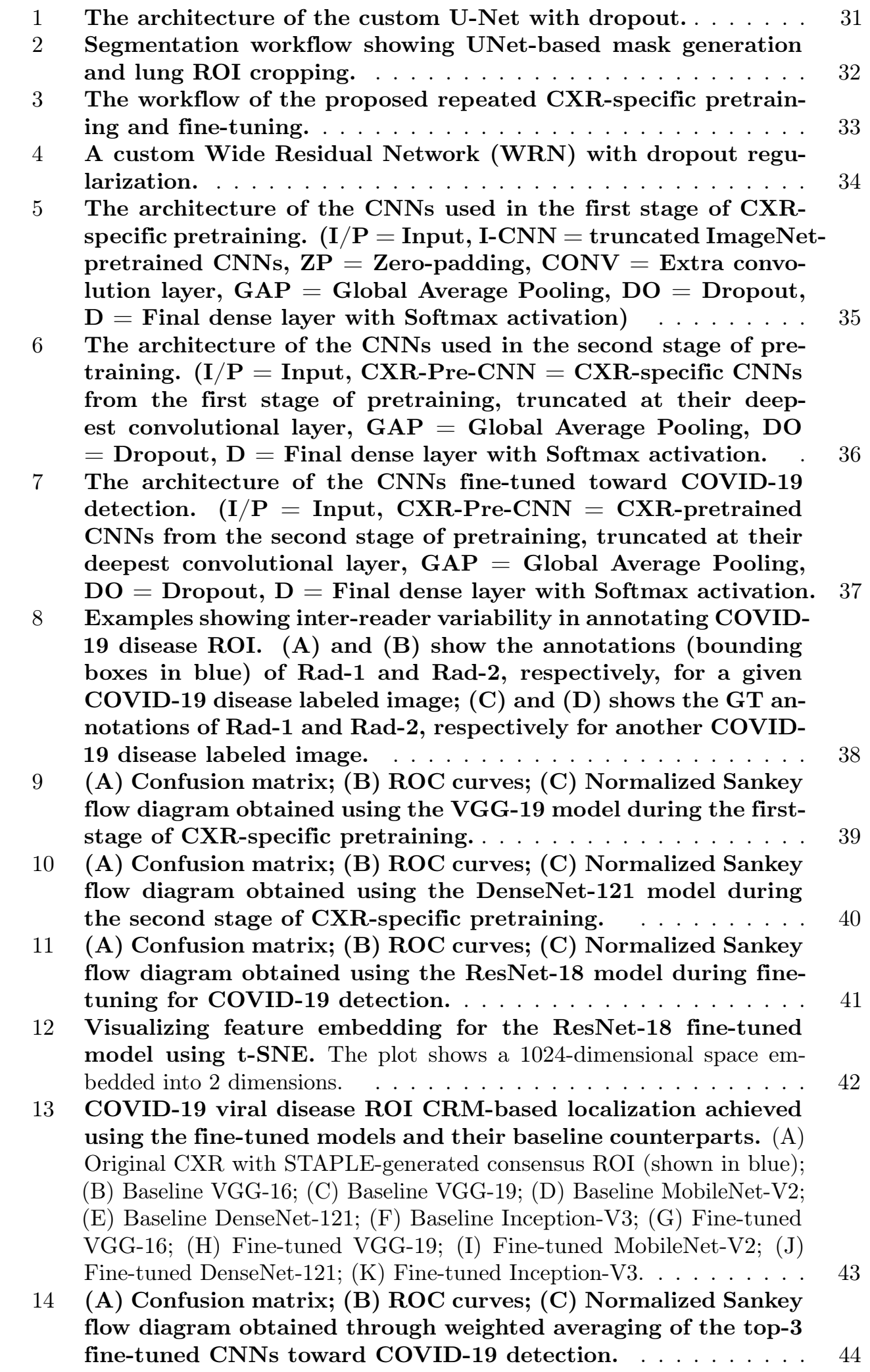


$15 \quad$ PR curves for the top-performing models using annotations of (A) Rad-1; (B) Rad-2; (C) STAPLE-generated ROI consensus.

$16 \quad$ PR curves for the top-N performing models using annotations of (A) Rad-1 ( $\mathrm{N}=3)$; (B) Rad-2 ( $\mathrm{N}=5)$; (C) STAPLE-generated consensus ROI $(\mathbf{N}=\mathbf{3}) . \ldots \ldots \ldots \ldots \ldots \ldots$

17 Sample CXRs showing annotations generated by Rad-1 (A) and (E) (in blue); Rad-2 (B) and (F) (in green); (C) and (G) top-3 ensemble using STAPLE-generated consensus ROI (program) (in yellow); (D) and (H) STAPLE-generated consensus ROI annotation (in red). . . . . . . . . . . . . . . . . . 47

18 Instances of ensemble CRMs combining (A) top-3 CNNs using STAPLE-generated consensus ROI annotation; (B ) top-5 CNNs using Rad-2 annotations. The green box denotes reference ROI annotation and the blue box denotes ensemble CRM

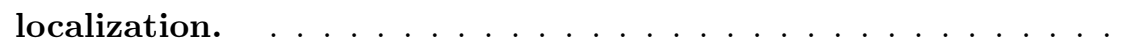
48

19 (A) Mean plot for the mAP scores obtained by the top-N ensembles using Rad-1, Rad-2, and STAPLE-generated consensus ROI annotations; Error bars represent standard errors. The differences are not statistically significant; (B) Residual plot \begin{tabular}{ll|lll} 
showing the data follow the normal distribution & $\ldots$ & $\ldots$ & $\ldots$ & 49
\end{tabular}

20 Assessing inter-reader variability and program performance.The following performance metrics are measured and plotted for 10 different IoU thresholds in the range $(0.1-0.7)$ : (A) Kappa statistic; (B) Sensitivity; (C) Specificity; (D) PPV. $\ldots \ldots \ldots \ldots \ldots \ldots$ 


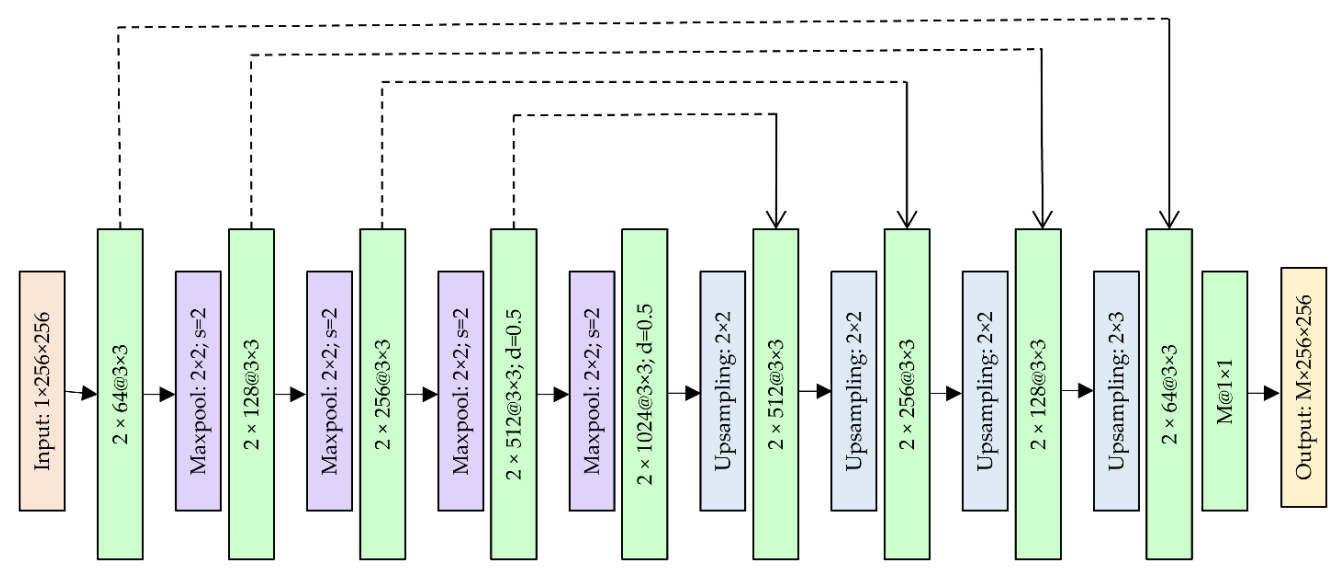

Fig 1. The architecture of the custom U-Net with dropout. 
medRxiv preprint doi: https://doi.org/10.1101/2020.07.15.20154385; this version posted July 16, 2020. The copyright holder for this preprint (which was not certified by peer review) is the author/funder, who has granted medRxiv a license to display the preprint in perpetuity.

\section{It is made available under a CC-BY-NC-ND 4.0 International license .}

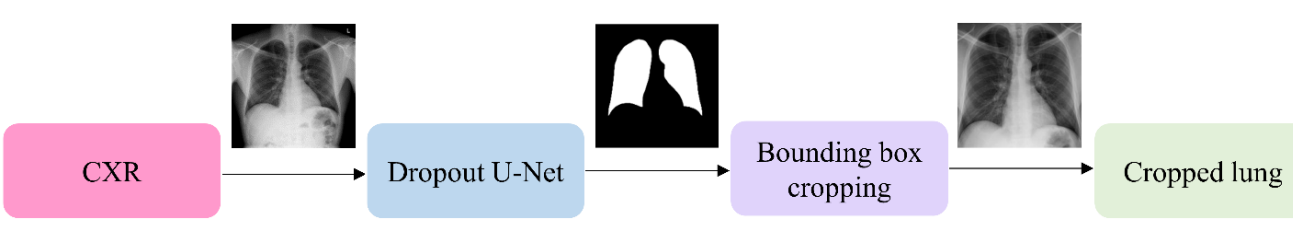

Fig 2. Segmentation workflow showing UNet-based mask generation and lung ROI cropping. 
medRxiv preprint doi: https://doi.org/10.1101/2020.07.15.20154385; this version posted July 16, 2020. The copyright holder for this preprint (which was not certified by peer review) is the author/funder, who has granted medRxiv a license to display the preprint in perpetuity.

\section{It is made available under a CC-BY-NC-ND 4.0 International license .}

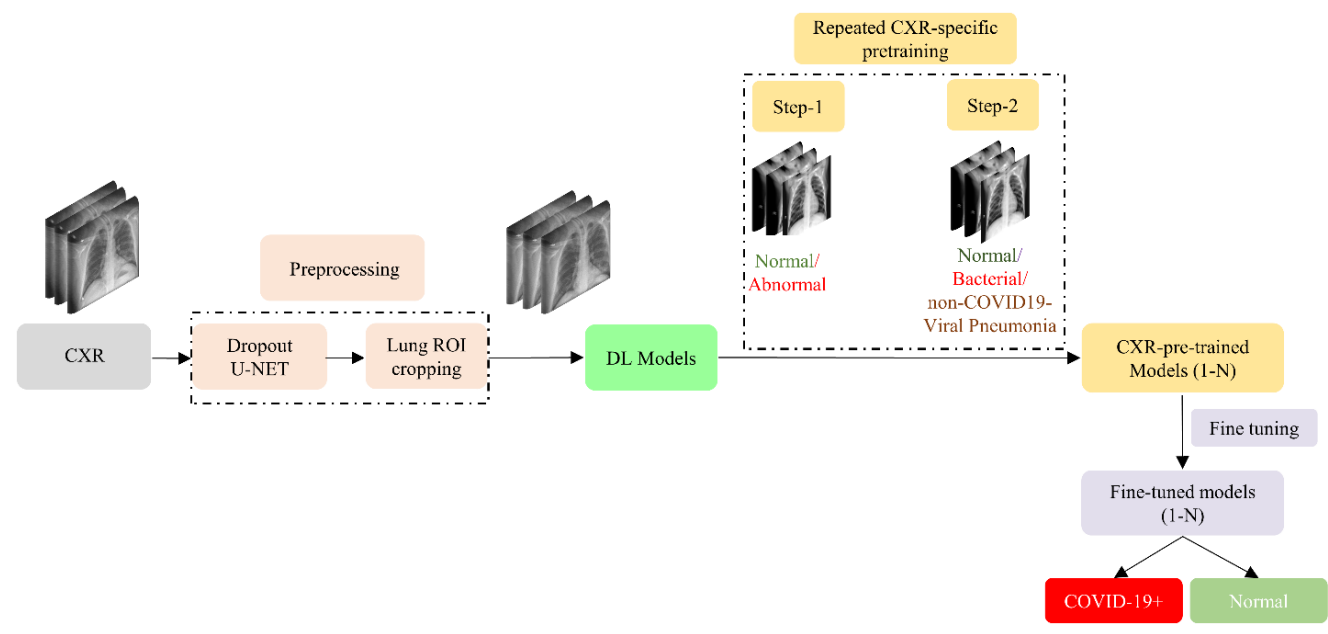

Fig 3. The workflow of the proposed repeated CXR-specific pretraining and fine-tuning. 
medRxiv preprint doi: https://doi.org/10.1101/2020.07.15.20154385; this version posted July 16, 2020. The copyright holder for this preprint (which was not certified by peer review) is the author/funder, who has granted medRxiv a license to display the preprint in perpetuity.

\section{It is made available under a CC-BY-NC-ND 4.0 International license .}

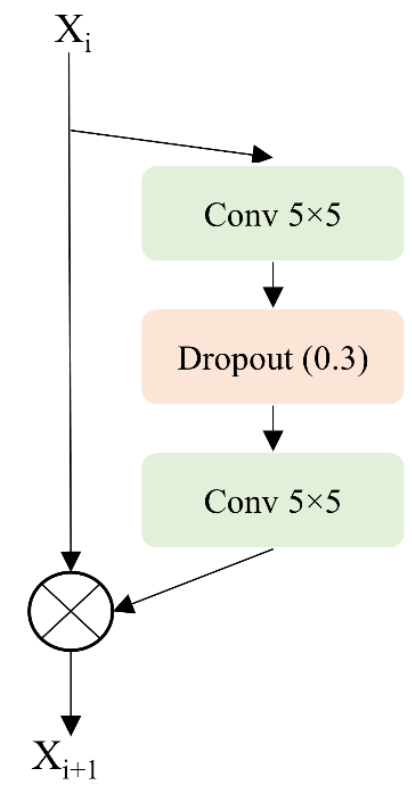

Fig 4. A custom Wide Residual Network (WRN) with dropout regularization. 
medRxiv preprint doi: https://doi.org/10.1101/2020.07.15.20154385; this version posted July 16, 2020. The copyright holder for this preprint (which was not certified by peer review) is the author/funder, who has granted medRxiv a license to display the preprint in perpetuity. It is made available under a CC-BY-NC-ND 4.0 International license .
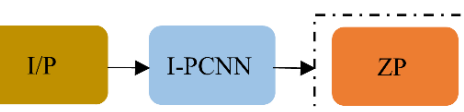

GAP

DO
Normal

Fig 5. The architecture of the CNNs used in the first stage of CXR-specific pretraining. $(\mathrm{I} / \mathrm{P}=$ Input, I-CNN $=$ truncated ImageNet-pretrained CNNs, ZP = Zero-padding, CONV = Extra convolution layer, GAP = Global Average Pooling, DO $=$ Dropout, $\mathbf{D}=$ Final dense layer with Softmax activation) 
medRxiv preprint doi: https://doi.org/10.1101/2020.07.15.20154385; this version posted July 16, 2020. The copyright holder for this preprint (which was not certified by peer review) is the author/funder, who has granted medRxiv a license to display the preprint in perpetuity.

\section{It is made available under a CC-BY-NC-ND 4.0 International license .}

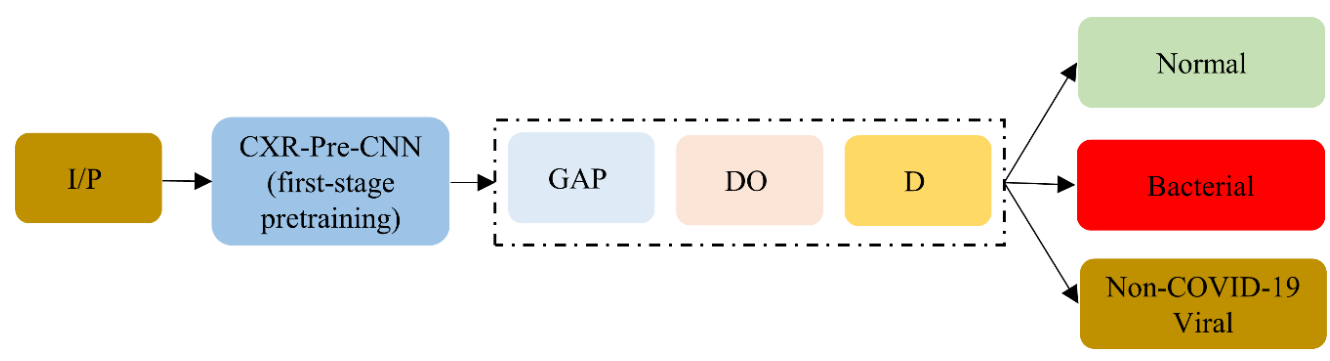

Fig 6. The architecture of the CNNs used in the second stage of pretraining. (I/P $=$ Input, CXR-Pre-CNN $=$ CXR-specific CNNs from the first stage of pretraining, truncated at their deepest convolutional layer, GAP = Global Average Pooling, DO = Dropout, D = Final dense layer with Softmax activation. 
medRxiv preprint doi: https://doi.org/10.1101/2020.07.15.20154385; this version posted July 16, 2020. The copyright holder for this preprint (which was not certified by peer review) is the author/funder, who has granted medRxiv a license to display the preprint in perpetuity.

It is made available under a CC-BY-NC-ND 4.0 International license.

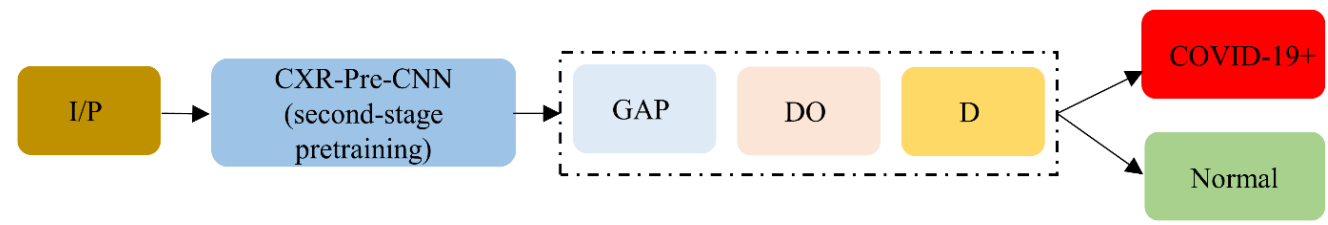

Fig 7. The architecture of the CNNs fine-tuned toward COVID-19 detection. (I/P = Input, CXR-Pre-CNN = CXR-pretrained CNNs from the second stage of pretraining, truncated at their deepest convolutional layer, GAP $=$ Global Average Pooling, $\mathbf{D O}=$ Dropout, $\mathbf{D}=$ Final dense layer with Softmax activation. 
medRxiv preprint doi: https://doi.org/10.1101/2020.07.15.20154385; this version posted July 16, 2020. The copyright holder for this preprint (which was not certified by peer review) is the author/funder, who has granted medRxiv a license to display the preprint in perpetuity.

It is made available under a CC-BY-NC-ND 4.0 International license .

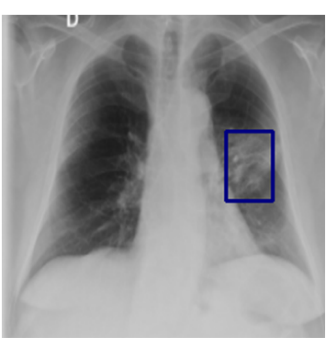

(A)

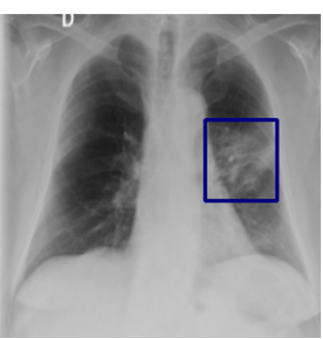

(B)

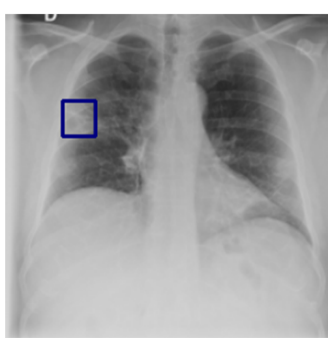

(C)



(D)

Fig 8. Examples showing inter-reader variability in annotating COVID-19 disease ROI. (A) and (B) show the annotations (bounding boxes in blue) of Rad-1 and Rad-2, respectively, for a given COVID-19 disease labeled image; (C) and (D) shows the GT annotations of Rad-1 and Rad-2, respectively for another COVID-19 disease labeled image. 
medRxiv preprint doi: https://doi.org/10.1101/2020.07.15.20154385; this version posted July 16,2020 . The copyright holder for this preprint (which was not certified by peer review) is the author/funder, who has granted medRxiv a license to display the preprint in perpetuity.

\section{It is made available under a CC-BY-NC-ND 4.0 International license.}

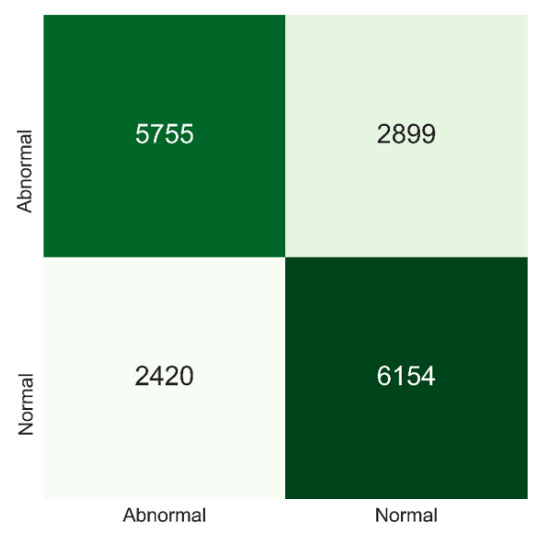

$(A)$

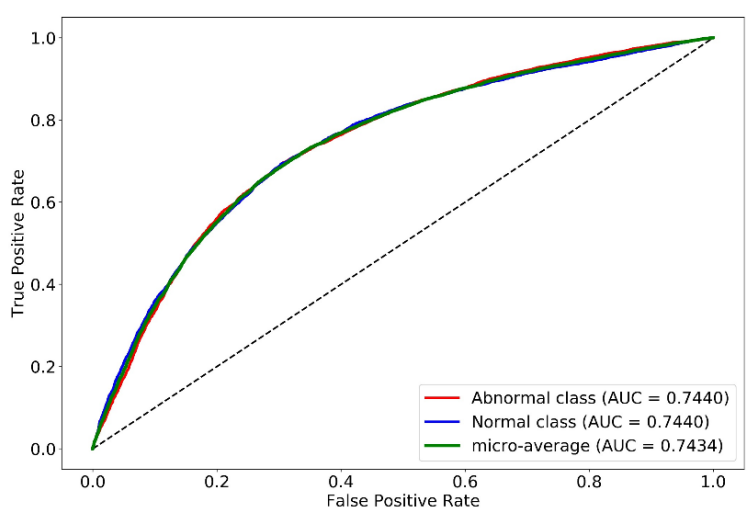

(B)

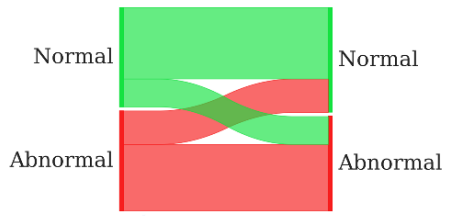

(C)

Fig 9. (A) Confusion matrix; (B) ROC curves; (C) Normalized Sankey flow diagram obtained using the VGG-19 model during the first-stage of CXR-specific pretraining. 
medRxiv preprint doi: https://doi.org/10.1101/2020.07.15.20154385; this version posted July 16, 2020. The copyright holder for this preprint (which was not certified by peer review) is the author/funder, who has granted medRxiv a license to display the preprint in perpetuity.

\section{It is made available under a CC-BY-NC-ND 4.0 International license .}



(A)

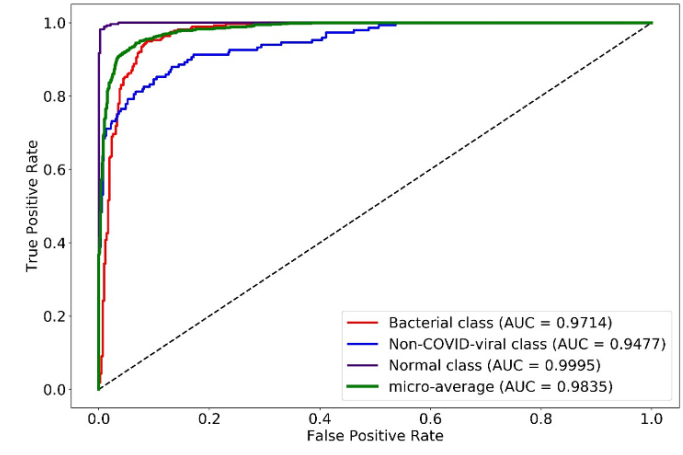

(B)

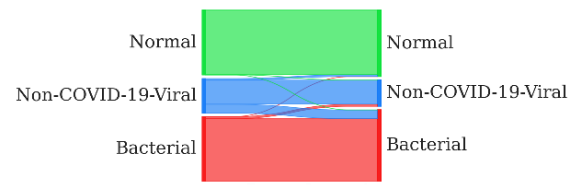

(C)

Fig 10. (A) Confusion matrix; (B) ROC curves; (C) Normalized Sankey flow diagram obtained using the DenseNet-121 model during the second stage of CXR-specific pretraining. 
medRxiv preprint doi: https://doi.org/10.1101/2020.07.15.20154385; this version posted July 16,2020 . The copyright holder for this preprint (which was not certified by peer review) is the author/funder, who has granted medRxiv a license to display the preprint in perpetuity.

\section{It is made available under a CC-BY-NC-ND 4.0 International license .}

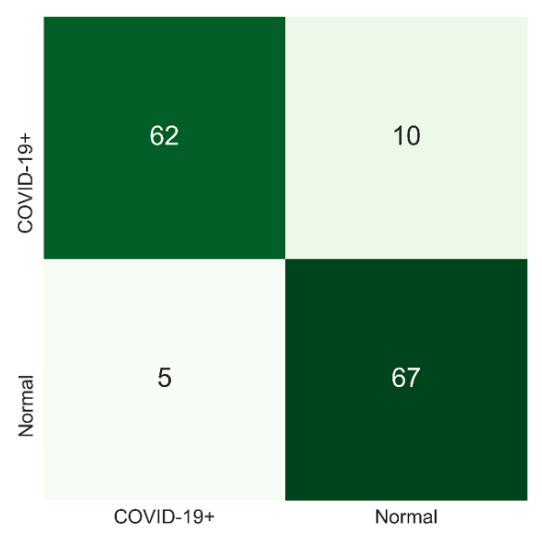

(A)

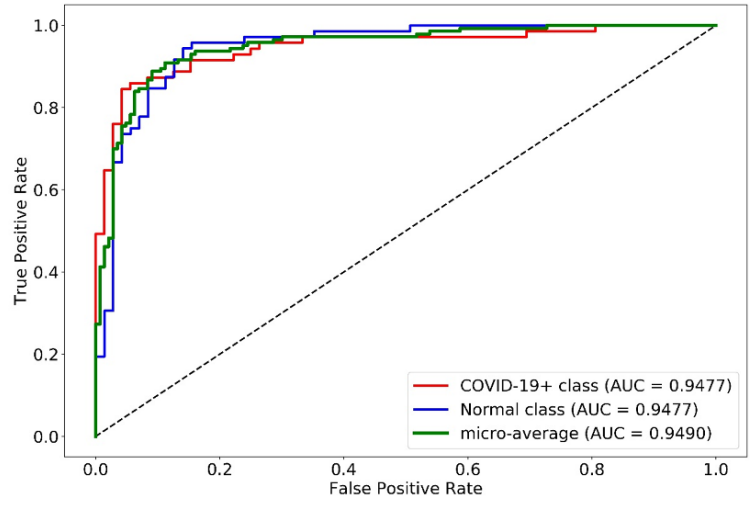

(B)

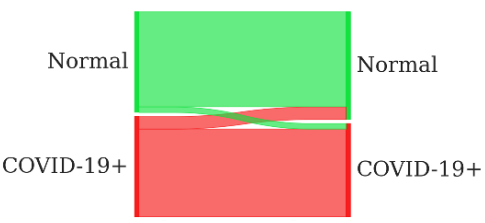

(C)

Fig 11. (A) Confusion matrix; (B) ROC curves; (C) Normalized Sankey flow diagram obtained using the ResNet-18 model during fine-tuning for COVID-19 detection. 

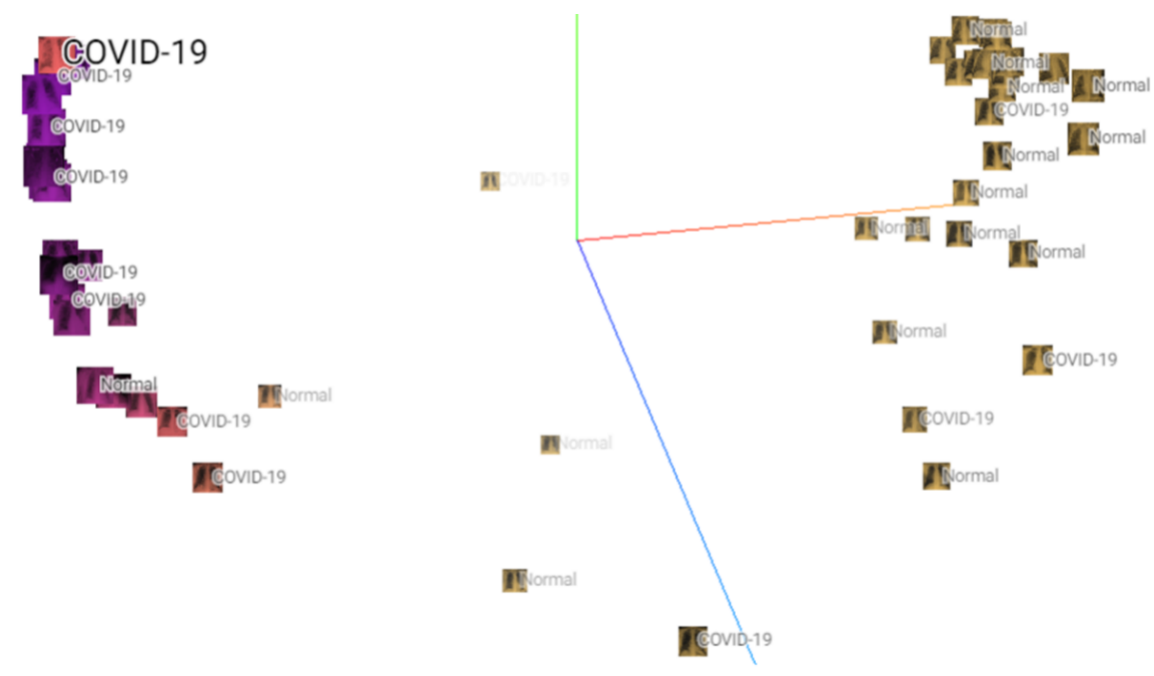

Fig 12. Visualizing feature embedding for the ResNet-18 fine-tuned model using t-SNE. The plot shows a 1024-dimensional space embedded into 2 dimensions. 


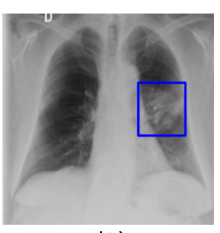

(A)

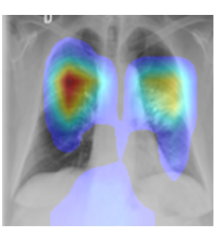

(B)

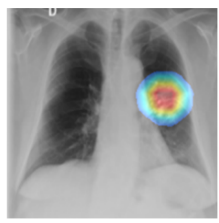

(G)



(C)



(H)

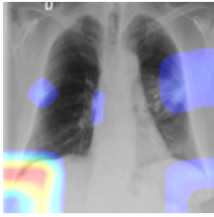

(D)

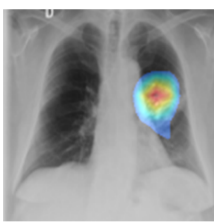

(I)

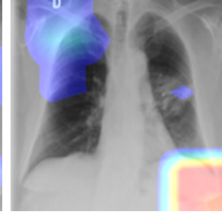

(E)

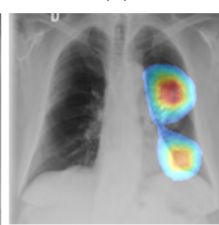

(J)

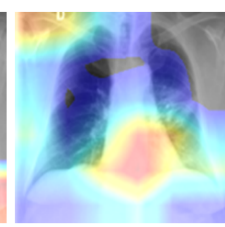

(F)

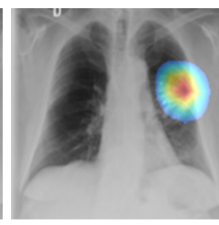

(K)

Fig 13. COVID-19 viral disease ROI CRM-based localization achieved using the fine-tuned models and their baseline counterparts. (A) Original CXR with STAPLE-generated consensus ROI (shown in blue); (B) Baseline VGG-16; (C) Baseline VGG-19; (D) Baseline MobileNet-V2; (E) Baseline DenseNet-121; (F) Baseline Inception-V3; (G) Fine-tuned VGG-16; (H) Fine-tuned VGG-19; (I) Fine-tuned MobileNet-V2; (J) Fine-tuned DenseNet-121; (K) Fine-tuned Inception-V3. 
medRxiv preprint doi: https://doi.org/10.1101/2020.07.15.20154385; this version posted July 16, 2020. The copyright holder for this preprint (which was not certified by peer review) is the author/funder, who has granted medRxiv a license to display the preprint in perpetuity.

\section{It is made available under a CC-BY-NC-ND 4.0 International license.}

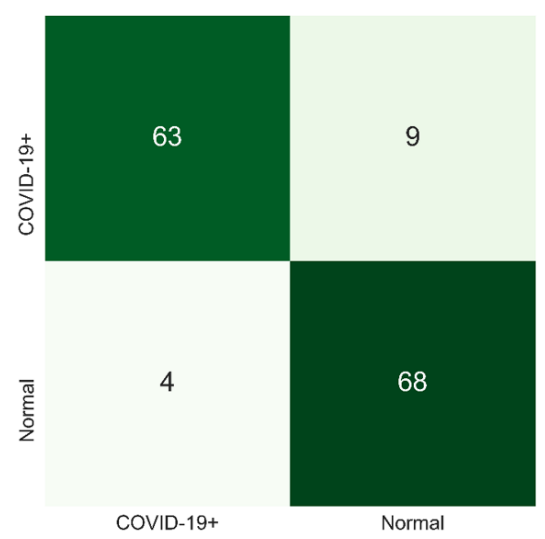

(A)

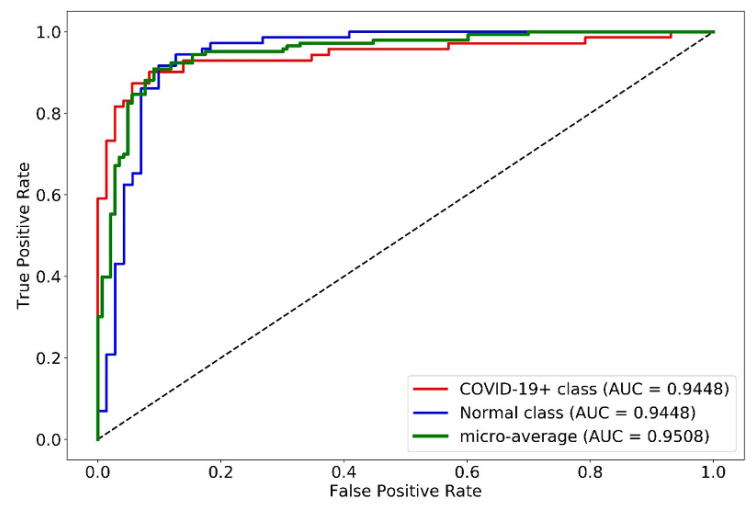

(B)

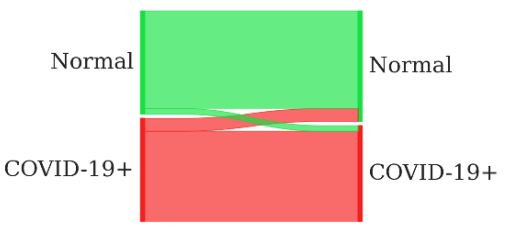

(C)

Fig 14. (A) Confusion matrix; (B) ROC curves; (C) Normalized Sankey flow diagram obtained through weighted averaging of the top-3 fine-tuned CNNs toward COVID-19 detection. 
medRxiv preprint doi: https://doi.org/10.1101/2020.07.15.20154385; this version posted July 16, 2020. The copyright holder for this preprint (which was not certified by peer review) is the author/funder, who has granted medRxiv a license to display the preprint in perpetuity.

\section{It is made available under a CC-BY-NC-ND 4.0 International license .}

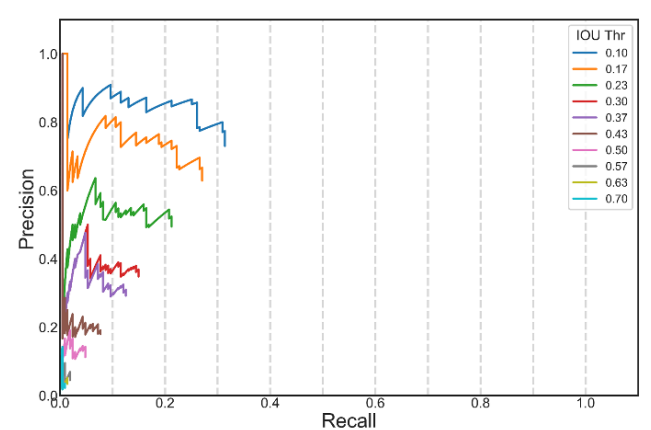

(A)

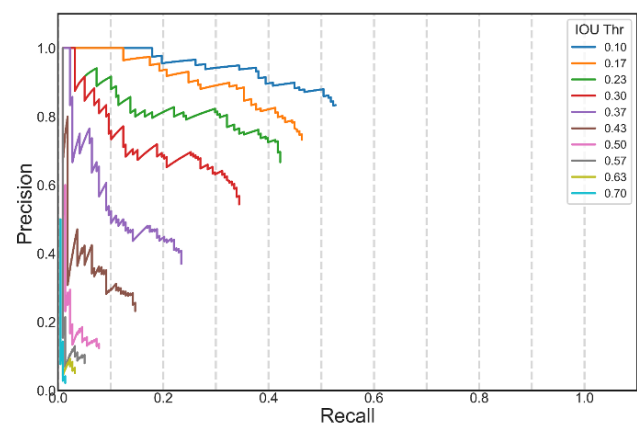

(B)

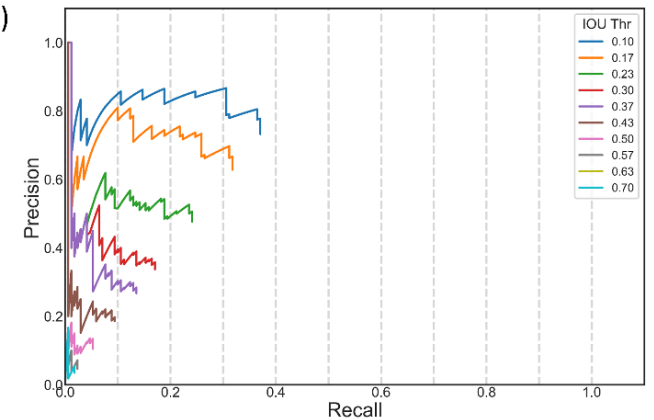

(C)

Fig 15. PR curves for the top-performing models using annotations of (A) Rad-1; (B) Rad-2; (C) STAPLE-generated ROI consensus. 
medRxiv preprint doi: https://doi.org/10.1101/2020.07.15.20154385; this version posted July 16, 2020. The copyright holder for this preprint (which was not certified by peer review) is the author/funder, who has granted medRxiv a license to display the preprint in perpetuity.

\section{It is made available under a CC-BY-NC-ND 4.0 International license .}
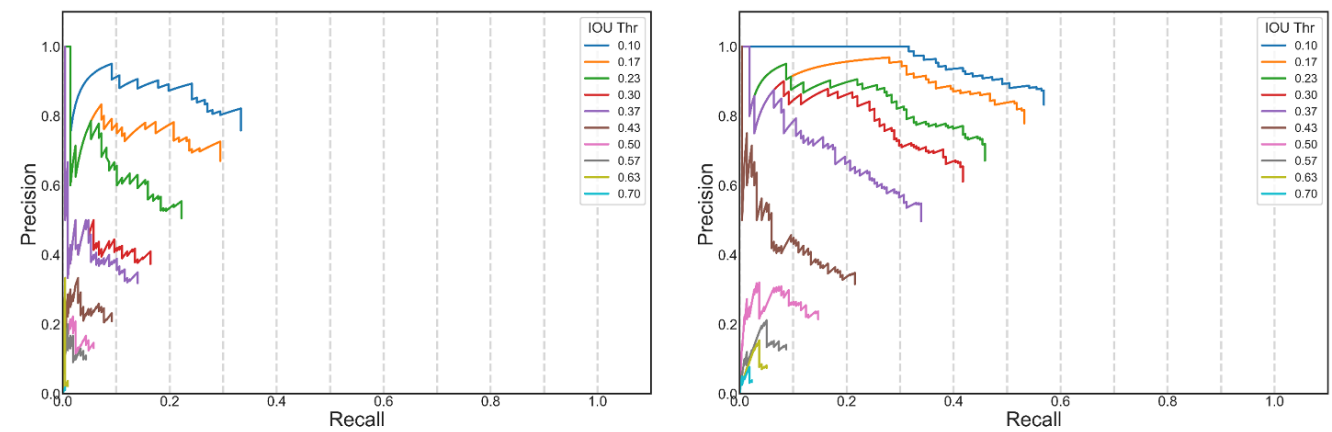

(A)

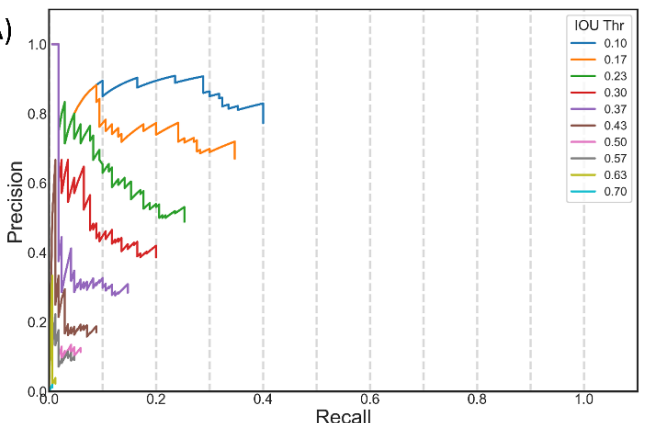

(B)

(C)

Fig 16. PR curves for the top-N performing models using annotations of (A) Rad-1 ( $\mathrm{N}=3)$; (B) Rad-2 $(\mathrm{N}=5)$; (C) STAPLE-generated consensus ROI $(\mathrm{N}=3)$. 


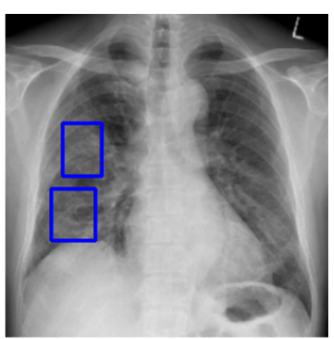

(A)

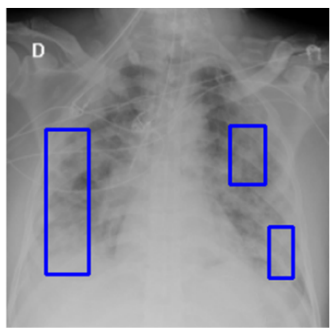

(E)

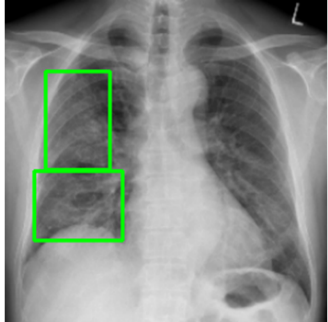

(B)

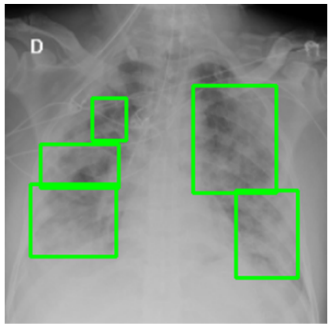

(F)



(C)

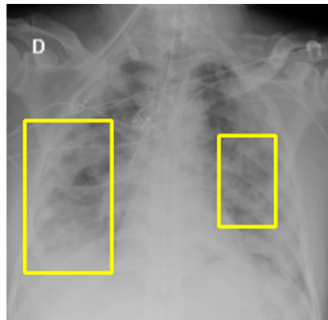

(G)

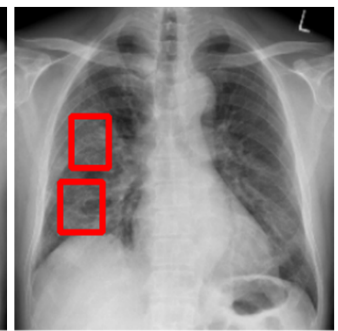

(D)

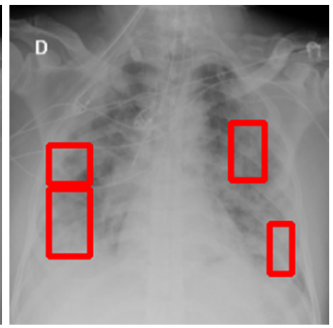

(H)

Fig 17. Sample CXRs showing annotations generated by Rad-1 (A) and (E) (in blue); Rad-2 (B) and (F) (in green); (C) and (G) top-3 ensemble using STAPLE-generated consensus ROI (program) (in yellow); (D) and (H) STAPLE-generated consensus ROI annotation (in red). 
medRxiv preprint doi: https://doi.org/10.1101/2020.07.15.20154385; this version posted July 16, 2020. The copyright holder for this preprint (which was not certified by peer review) is the author/funder, who has granted medRxiv a license to display the preprint in perpetuity.

It is made available under a CC-BY-NC-ND 4.0 International license .

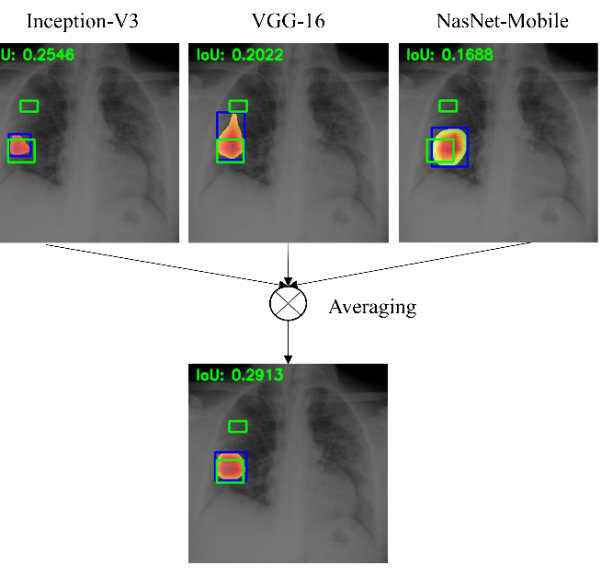

(A)

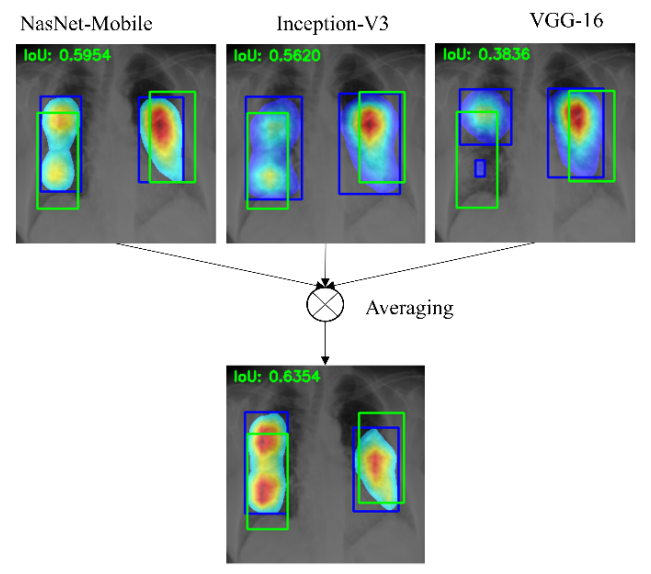

(B)

Fig 18. Instances of ensemble CRMs combining (A) top-3 CNNs using STAPLE-generated consensus ROI annotation; (B ) top-5 CNNs using Rad-2 annotations. The green box denotes reference ROI annotation and the blue box denotes ensemble CRM localization. 


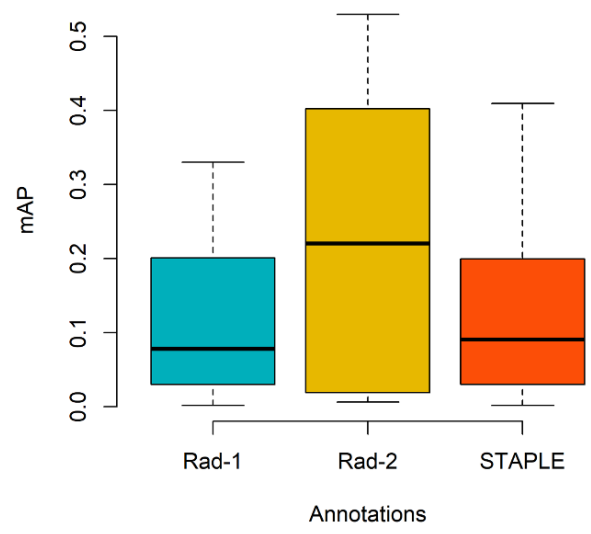

(A)



(B)

Fig 19. (A) Mean plot for the mAP scores obtained by the top-N ensembles using Rad-1, Rad-2, and STAPLE-generated consensus ROI annotations; Error bars represent standard errors. The differences are not statistically significant; (B) Residual plot showing the data follow the normal distribution . 




(A)

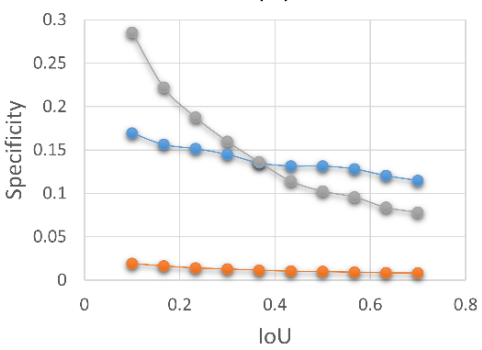

(C)

$-\operatorname{Rad}-1$

$\rightarrow-\operatorname{Rad}-2$

$\rightarrow$ Program

$\rightarrow$ Rad - 1

$\rightarrow$ Rad - 2

-Program

$$
0.8
$$$$
\text { (1) }
$$

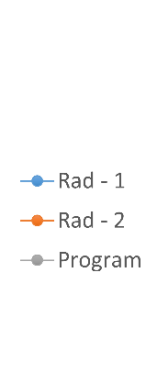

Fig 20. Assessing inter-reader variability and program performance. The following performance metrics are measured and plotted for 10 different IoU thresholds in the range $(0.1-0.7)$ : (A) Kappa statistic; (B) Sensitivity; (C) Specificity; (D) PPV.

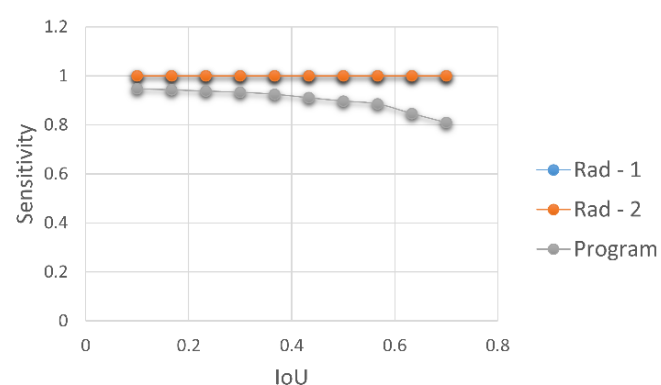

(B)

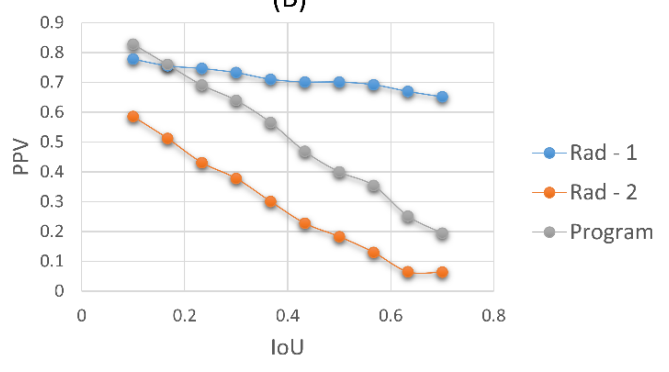

(D) 